\title{
Measurement of the scalar third-order electric polarizability of the Cs ground state using coherent-population-trapping spectroscopy in Ramsey geometry
}

\author{
Jean-Luc Robyr, ${ }^{*}$ Paul Knowles, ${ }^{\dagger}$ and Antoine Weis \\ Fribourg Atomic Physics Group, Department of Physics, University of Fribourg, Switzerland
}

(Received 24 February 2014; published 7 July 2014)

\begin{abstract}
The ac-Stark shift induced by blackbody radiation is a major source of systematic uncertainty in present-day cesium microwave frequency standards. The shift is parametrized in terms of a third-order electric polarizability $\alpha_{0}^{(3)}$ that can be inferred from the static electric-field displacement of the clock transition resonance. In this paper, we report on an all-optical coherent-population-trapping pump-probe experiment measuring the differential polarizability $\Delta \alpha_{0}^{(3)}=\alpha_{0}^{(3)}(F=4)-\alpha_{0}^{(3)}(F=3)$ on a thermal Cs atomic beam, from which we infer $\alpha_{0}^{(3)}(F=4)=2.023(6)_{\text {stat }}(9)_{\text {syst }} \mathrm{Hz} /(\mathrm{kV} / \mathrm{cm})^{2}$, which corresponds to a scalar Stark shift parameter $k_{s}=$ $-2.312(7)_{\text {stat }}(10)_{\text {syst }} \mathrm{Hz} /(\mathrm{kV} / \mathrm{cm})^{2}$. The result agrees within two standard deviations with a recent measurement in an atomic fountain, and rules out another recent result obtained in a Cs vapor cell.
\end{abstract}

DOI: 10.1103/PhysRevA.90.012505

PACS number(s): 32.10.Dk, 32.60.+i, 31.15.ap

\section{INTRODUCTION}

Blackbody radiation (BBR) displaces, via the ac-Stark shift, the Cs microwave clock transition frequency. This effect is a dominant limitation in the accuracy of present microwave atomic frequency standards at the $10^{-16}$ level $[1,2]$. Corrections of the clock frequency's BBR shift rely either on a precise and accurate knowledge of the electric polarizabilities describing the shift (a contemporary, although not exhaustive, review of atomic polarizabilities is given by Mitroy et al. [3]) or on a direct frequency comparison between primary frequency standards working at different temperatures. The most accurate measurement of the relevant parameter, expressed as a difference of polarizabilities $\Delta \alpha_{0}^{(3)}$ (defined below), has been extracted from a Stark shift measurement in dc electric field [4]. We also mention a recent accurate direct measurement of the Stark frequency shift between three Cs primary frequency standards that has reached an uncertainty below $1 \%$ [5]. The motivation for the work herein arose from the $6 \sigma$ disaccord between the two most recent measurements of $\Delta \alpha_{0}^{(3)}: 4.564(8) \mathrm{Hz} /(\mathrm{kV} / \mathrm{cm})^{2}[4]$ and $4.10(8) \mathrm{Hz} /(\mathrm{kV} / \mathrm{cm})^{2}$ [6]. The work of [4], which built on the work of [7], measured the shift using a static electric field applied to atoms in an atomic fountain clock, whereas the work of [6] used a static field applied (externally) to an atomic Cs vapor confined in a glass cell. We note that the result [4] is in agreement with recent theoretical values [8-10].

In this work, we present an alternative experimental approach for measuring $\Delta \alpha_{0}^{(3)}$. An atomic beam technique was adapted to an all-optical pump-probe experiment using coherent population trapping (CPT), both to create a $\Delta m_{F}=0$ hyperfine coherence in the Cs ground state and to subsequently probe the coherence following its evolution in applied static electric and magnetic fields. The resulting Ramsey resonance data were analyzed in two complementary ways: first by

\footnotetext{
*Present address: SYRTE-Observatoire de Paris, 75014 Paris, France; Jean-Luc.Robyr@obspm.fr

${ }^{\dagger}$ Present address: Rilkeplatz 8/9, A-1040 Vienna, Austria.

${ }^{\ddagger}$ http://physics.unifr.ch/en/page/89/
}

a Fourier decomposition method applied to scans of the fringe pattern $[11,12]$, and second by tracking the central fringe's zero crossing, with both measured as a function of the applied electric field's magnitude. In the following, we present the conventional parametrization of the effect, develop the signal model and its analysis by Fourier decomposition, introduce pertinent details of the apparatus, and give the analysis and results, including a discussion on limiting systematics.

\section{THEORY}

\section{A. The Stark shift}

The interaction of an atom with an applied electric field $\overrightarrow{\mathcal{E}}$ is described by the Stark Hamiltonian $\mathbb{H}_{\mathrm{St}}=-\vec{d} \cdot \overrightarrow{\mathcal{E}}$. The energy shift $\Delta E_{F, m_{F}}$ of a magnetic hyperfine sublevel $\left|n^{2} S_{1 / 2}, F, m_{F}\right\rangle$ of an alkali atom ground state is parametrized as $\Delta E_{F, m_{F}}=$ $-\frac{1}{2} \alpha|\overrightarrow{\mathcal{E}}|^{2}$, where the polarizability $\alpha$ is calculated by perturbation theory using the Hamiltonian $\mathbb{H}=\mathbb{H}_{\mathrm{St}}+\mathbb{H}_{\mathrm{hf}}$, with $\mathbb{H}_{\text {hf }}$ being the hyperfine interaction Hamiltonian. Transition energies between internal atomic levels will consequently change in proportion to the difference of the involved states' polarizabilities. The polarizability $\alpha$ is traditionally broken down via series expansion in both the perturbation order $n$ at which the component contributes and the multipole order $k$ of its interaction, which, following the notation and methods established in Refs. [13-16], we will denote as $\alpha_{k}^{(n)}$.

The sublevel energies of the Cs $6 S_{1 / 2}$ ground state of interest here are affected only by the polarizabilities $\alpha_{0}^{(2)}$, $\alpha_{0}^{(3)}$, and $\alpha_{2}^{(3)}$, where the (by far dominating) scalar secondorder polarizability $\alpha_{0}^{(2)}$ is independent of $F$ and $m_{F}$, and therefore does not contribute to a differential energy shift of the states coupled by the clock transition. The third-order scalar polarizability $\alpha_{0}^{(3)}$ depends only on $F$, while the thirdorder tensor polarizability $\alpha_{2}^{(3)}$ depends both on $F$ and $m_{F}$, so that both values affect the clock transition's dc Stark shift. The relevant $\alpha$ 's of the $F=3$ state are expressible as constants times the polarizabilities of the $F=4$ state, so that the electric-field-induced frequency shift of the $\Delta m_{F}=0$ 
transitions $\left|6 S_{1 / 2}, F=3, m_{F}\right\rangle \rightarrow\left|6 S_{1 / 2}, F=4, m_{F}\right\rangle$ can be expressed as

$$
\begin{aligned}
\Delta v_{\text {Stark }}\left(m_{F}\right) & =-\frac{1}{2}\left[\frac{16}{7} \alpha_{0}^{(3)}+\frac{3 m_{F}^{2}-16}{28} f(\theta) \alpha_{2}^{(3)}\right]|\overrightarrow{\mathcal{E}}|^{2} \\
& \equiv-\frac{1}{2} \alpha\left(m_{F}\right) \mathcal{E}^{2}
\end{aligned}
$$

where $\alpha_{0}^{(3)}=\alpha_{0}^{(3)}(F=4)$ and $\alpha_{2}^{(3)}=\alpha_{2}^{(3)}(F=4)$ are the thirdorder scalar and tensor polarizabilities, respectively, of the $F=$ 4 hyperfine state, $m_{F}$ is the magnetic quantum number defined by the quantization axis (chosen along the magnetic field $\hat{\mathcal{B}}$ ), and $f(\theta)=3 \cos ^{2}(\theta)-1$ with $\cos \theta=\hat{\mathcal{E}} \cdot \hat{\mathcal{B}}$. Equations (1a) and (1b) represent the result originally derived by Sandars [17], after correction of a sign error that was uncovered in Refs. $[15,16]$. Note also that we set $h=1$ in the definition of polarizabilities, so that the latter are expressed in the practical "laboratory units" of $\mathrm{Hz} /(\mathrm{kV} / \mathrm{cm})^{2}$.

The Stark shift, $\Delta v_{\text {Stark }}\left(m_{F}=0\right)$, of the clock transition $\left|6 S_{1 / 2}, F=3, m_{F}=0\right\rangle \rightarrow\left|6 S_{1 / 2}, F=4, m_{F}=0\right\rangle$ can be parametrized as

$$
\Delta v_{\text {Stark }}\left(m_{F}=0\right)=\left[k_{s}+k_{t} f(\theta)\right]|\overrightarrow{\mathcal{E}}|^{2},
$$

with scalar and tensor constants $k_{s}$ and $k_{t}$ that are related to the quantities introduced above via

$$
k_{s}+k_{t} f(\theta)=-\frac{8}{7} \alpha_{0}^{(3)}+\frac{2}{7} \alpha_{2}^{(3)} f(\theta) .
$$

The clock shift arising from the blackbody spectrum is isotropic, implying $\langle f(\theta)\rangle=0$, and is thus not sensitive to $\alpha_{2}^{(3)}$, i.e., to $k_{t}$. Laboratory experiments, on the other hand, measuring the clock shift by applied ac or dc fields must, therefore, consider $\alpha_{2}^{(3)}$ (or $k_{t}$ ) when extracting $\alpha_{0}^{(3)}$ (or $k_{s}$ ) from the measured effect, while model calculations of the BBR frequency shift determine the scalar contribution $k_{s}[9,10]$ only.

The dependence of the BBR shift on temperature $T$ is normally [1] expressed in one of three ways,

$$
\begin{aligned}
\delta v(T) & =\frac{-\Delta \alpha_{0}^{(3)}}{2}\left(831.9 \frac{\mathrm{V}}{\mathrm{m}}\right)^{2}\left(\frac{T}{300 \mathrm{~K}}\right)^{4}\left[1+\epsilon\left(\frac{T}{300 \mathrm{~K}}\right)^{2}\right] \\
& =k_{s}\left(831.9 \frac{\mathrm{V}}{\mathrm{m}}\right)^{2}\left(\frac{T}{300 \mathrm{~K}}\right)^{4}\left[1+\epsilon\left(\frac{T}{300 \mathrm{~K}}\right)^{2}\right], \\
& =\beta v_{00}\left(\frac{T}{300 \mathrm{~K}}\right)^{4}\left[1+\epsilon\left(\frac{T}{300 \mathrm{~K}}\right)^{2}\right],
\end{aligned}
$$

where $v_{00}=9192631770 \mathrm{~Hz}$ is the Cs clock transition frequency and $831.9 \mathrm{~V} / \mathrm{m}$ represents the root-mean-square (rms) electric field of the blackbody spectrum at $300 \mathrm{~K}$. The correction factor $\epsilon$ was evaluated to be 0.014 [1] or 0.013 [18]. Various publications present either $\alpha_{0}^{(3)}(F), \Delta \alpha_{0}^{(3)}\left[\equiv \alpha_{0}^{(3)}(F=\right.$ $\left.4)-\alpha_{0}^{(3)}(F=3)=+\frac{16}{7} \alpha_{0}^{(3)}(F=4)\right], k_{s}\left[=-\frac{8}{7} \alpha_{0}^{(3)}(F=4)\right]$, or $\beta$.

\section{B. Experimental approach}

Experiments were carried out in an effusive cesium beam using an all-optical Ramsey pump-probe technique relying on coherent population trapping (CPT) by a bichromatic laser field, rather than on the conventional Ramsey resonance method using (spatially or temporally) separated interactions

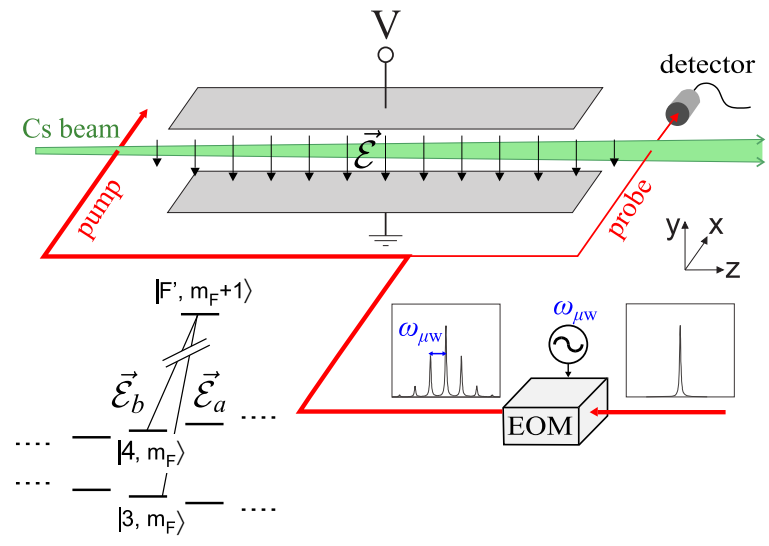

FIG. 1. (Color online) Principle of the all-optical Ramsey method for measuring the Stark shift. The carrier and a sideband (spaced by $\omega_{\mu \mathrm{w}}$ ) of a phase-modulated laser beam are used to create a coherent superposition of ground-state hyperfine levels in a Cs atomic beam. The phase accumulated by the coherence through the atoms' interaction with combined electric and magnetic fields is probed by a weaker bichromatic field whose microwave modulation has a fixed, but tunable phase with respect to the pump field modulation.

in microwave cavities, such as deployed in atomic beams $[19,20]$ or in fountain clocks [4]. Details of our method were already described in the literature [21-23]. In brief, CPT pumping by a phase-coherent bichromatic optical field with components of identical (circular or linear) polarization and with frequency splitting given by the microwave frequency $\omega_{\mu \mathrm{w}}$ is used to create a specific coherent superposition of hyperfine-Zeeman states $\left|F=3, m_{F}\right\rangle$ and $\left|F=4, m_{F}\right\rangle$ in the Cs ground state (Fig. 1). In a subsequent light-free evolution zone, the coherence oscillates at the specific level splitting frequency $\omega_{0}$ that is subject to tunable static electric and magnetic fields. In a probe zone, the phase accumulated by the hyperfine coherence is compared to the phase accumulated by the constant evolution of the transition-driving microwave oscillator. When $\omega_{\mu \mathrm{w}}$ is scanned, Ramsey resonance signals are observed in the transmitted probe laser power. When a magnetic field lifts the Zeeman degeneracy, the full spectrum contains six or seven distinct $\Delta m_{F}=0$ resonances depending on the relative orientation of the magnetic and electric fields and the light fields' polarization.

We have implemented two different methods of data collection and analysis. In the first method, full Ramsey resonance curves were measured by scanning $\omega_{\mu \mathrm{w}}$ for different electric-field values and the data were reduced by Fourier decomposition.

In the second method, active feedback was used to lock the frequency $\omega_{\mu \mathrm{w}}$ of the microwave generator (producing the bichromatic laser field by phase modulation) to the zero crossing of the central dispersive Ramsey fringe, allowing a tracking of $\omega_{\mu \mathrm{w}}$ as a function of the applied electric-field strength.

The two methods have their respective advantages and drawbacks. The Fourier decomposition allows the extraction of both the Ramsey signal frequency and its phase at the cost of a slow acquisition rate, since $\omega_{\mu \mathrm{w}}$ has to be scanned several times over the Ramsey spectrum (20 s per scan). 
Conversely, recording the locked value of $\omega_{\mu \mathrm{w}}$ with respect to the central Ramsey fringe zero-crossing point yields a much better statistical uncertainty, since the signal of interest is continuously recorded, but has the disadvantage that the frequency and phase information are not separable.

\section{Atomic pump-evolution-probe model}

Consider the two hyperfine ground states $|a\rangle=$ $\left|6 S_{1 / 2}, F=3, m_{F}\right\rangle$ and $|b\rangle=\left|6 S_{1 / 2}, F=4, m_{F}\right\rangle$ (inset of Fig. 1) whose energies differ by the hyperfine splitting energy $\hbar \omega_{0}$. The bichromatic pump light field

$$
\begin{aligned}
\overrightarrow{\mathcal{E}}_{\text {pump }}(t) & =\overrightarrow{\mathcal{E}}_{a} e^{-i \omega_{a} t}+\overrightarrow{\mathcal{E}}_{b} e^{-i \omega_{b} t} \\
& =\overrightarrow{\mathcal{E}}_{a} e^{-i \omega t}+\overrightarrow{\mathcal{E}}_{b} e^{-i\left(\omega-\omega_{\mu \mathrm{w}}\right) t},
\end{aligned}
$$

where the fields $\overrightarrow{\mathcal{E}}_{a}$ and $\overrightarrow{\mathcal{E}}_{b}$ are near resonant with transitions $|a\rangle \rightarrow|e\rangle$ and $|b\rangle \rightarrow|e\rangle$ to a specific excited state $|e\rangle=$ $\left|6 P_{1 / 2}, F^{\prime}, m_{F}^{\prime}\right\rangle$, will put the atoms into a coherent superposition state,

$$
\left|\Psi_{0}\right\rangle=\eta_{a}|a\rangle+\eta_{b}|b\rangle e^{-i \omega_{0} t},
$$

where we have omitted the (irrelevant) phase factor $e^{-i \omega t}$. When probed by a weaker bichromatic field, in-phase with the pump field

$$
\overrightarrow{\mathcal{E}}_{\text {probe }}(t)=\varepsilon \overrightarrow{\mathcal{E}}_{a} e^{-i \omega t}+\varepsilon \overrightarrow{\mathcal{E}}_{b} e^{-i\left(\omega-\omega_{\mu \mathrm{w}}\right) t},
$$

where $\varepsilon$ is the ratio between the weak- and strong-field amplitudes, the power absorbed from the probe beam is given by

$$
\begin{aligned}
\Delta P_{\text {probe }} & \propto \varepsilon^{2}\left|\left\langle e\left|\vec{d} \cdot \overrightarrow{\mathcal{E}}_{\text {probe }}\right| \Psi_{0}\right\rangle\right|^{2} \\
& \propto\left|\eta_{a} \vec{d}_{a e} \cdot \overrightarrow{\mathcal{E}}_{a}+\eta_{b} \vec{d}_{b e} \cdot \overrightarrow{\mathcal{E}}_{b} e^{i\left(\omega_{\mu \mathrm{w}}-\omega_{0}\right) t}\right|^{2} \\
& \propto\left|\eta_{a} \Omega_{a e}+\eta_{b} \Omega_{b e} e^{i\left(\omega_{\mu \mathrm{w}}-\omega_{0}\right) t}\right|^{2}
\end{aligned}
$$

where $\vec{d}_{i e}$ denotes the electric dipole matrix elements $\langle e|\vec{d}| i\rangle$ and where the Rabi frequencies are defined by $\hbar \Omega_{i e}=\vec{d}_{i e} \cdot \overrightarrow{\mathcal{E}}_{i}$. On resonance, $\omega_{\mu \mathrm{w}}=\omega_{0}$, the absorbed power will vanish, when the state amplitudes $\eta_{a, b}$ obey

$$
\frac{\eta_{a}}{\eta_{b}}=-\frac{\Omega_{b e}}{\Omega_{a e}}=-\frac{\vec{d}_{b e} \cdot \overrightarrow{\mathcal{E}}_{b}}{\vec{d}_{a e} \cdot \overrightarrow{\mathcal{E}}_{a}}
$$

and the corresponding (normalized) state

$$
\left|\Psi_{0}\right\rangle=\frac{\Omega_{b e}}{\sqrt{\Omega_{a e}^{2}+\Omega_{b e}^{2}}}|a\rangle-\frac{\Omega_{a e}}{\sqrt{\Omega_{a e}^{2}+\Omega_{b e}^{2}}}|b\rangle e^{-i \omega_{0} t}
$$

is called a dark state, since it does not absorb light and hence does not emit fluorescence radiation. The formation of dark states, their perturbation by interactions with external fields, and their detection via light absorption (or fluorescence) form the basis of coherent-population-trapping (CPT) spectroscopy. With the matrix elements being given, the amplitudes $\mathcal{E}_{a, b}$ of the two field components can be chosen such that the state amplitudes in Eq. (12) obey $\eta_{a}=-\eta_{b}$, so that the power absorbed from the probe beam interrogating the atoms after an evolution time $T$ takes on the simple form

$$
\Delta P_{\text {probe }} \propto\left|1-e^{i\left(\omega_{\mu \mathrm{w}}-\omega_{0}\right) T}\right|^{2} \propto 1-\cos \left[\left(\omega_{0}-\omega_{\mu \mathrm{w}}\right) T\right] .
$$

\section{Phase and frequency shifts of the Ramsey spectrum}

The expressions above have assumed that the bichromatic fields in the pump and probe regions oscillate in phase. Since the pump and probe beams travel different paths from the source to their respective interaction zones, the relative phase between their two frequency components will acquire an additional spatial phase shift,

$$
\Delta \varphi_{\text {path }}=\omega_{\mu \mathrm{w}} \frac{\Delta x}{c},
$$

where $\Delta x$ is the difference of the paths traveled by the pump and probe beams. This phase propagates through the calculation and modifies the detuning-dependent term in Eq. (14) to

$$
\Delta P_{\text {probe }} \propto \cos \left[\left(\omega_{0}-\omega_{\mu \mathrm{w}}\right) T-\Delta \varphi_{\text {path }}\right] .
$$

Choosing the propagation phase to be $\Delta \varphi_{\text {path }}=0 \bmod \pi$ will yield symmetric (absorptive) Ramsey fringes with respect to the line center $\left(\omega_{\mu \mathrm{w}}=\omega_{0}\right)$, while $\Delta \varphi_{\text {path }}=\pi / 2 \bmod \pi$ will produce antisymmetric (dispersive) fringes. We define the Ramsey signal $S\left(\omega_{\mu \mathrm{w}}\right)$ as being the velocity-averaged change of the probe transmission (16) for a separation $L$ between the pump and the probe optical beams, viz.,

$$
\begin{aligned}
S\left(\omega_{\mu \mathrm{w}}\right)= & \int_{0}^{\infty} \rho(v) \cos \left\{\left[\omega_{0}+\Delta \omega\left(m_{F}\right)-\omega_{\mu \mathrm{w}}\right] \frac{L}{v}\right. \\
& \left.-\Delta \varphi\left(m_{F}\right)\right\} d v,
\end{aligned}
$$

where $\rho(v)$ is the atomic velocity distribution, and where the Stark and Zeeman shifts, i.e., the frequency shift induced by the electric and magnetic fields, respectively, are given by

$$
\Delta \omega\left(m_{F}\right)=\Delta \omega_{\text {Stark }}\left(m_{F}\right)+\Delta \omega_{\text {Zeeman }}\left(m_{F}\right),
$$

while

$$
\Delta \varphi\left(m_{F}\right)=\Delta \varphi_{\text {path }}\left(m_{F}\right)+\Delta \varphi_{\text {mot }}\left(m_{F}\right)
$$

represents the sum of phase shifts due to the pump and probe beams path-length differences and due to the motional Zeeman effects, respectively, as discussed in more detail below. For hyperfine coherences formed by the pair of states $\left|3, m_{F}\right\rangle$ and $\left|4, m_{F}\right\rangle$, the (differential) frequency shift induced by the Stark interaction of interest is $\Delta \omega_{\text {Stark }}=2 \pi \Delta v_{\text {Stark }}\left(m_{F}\right)$, where $\Delta v_{\text {Stark }}\left(m_{F}\right)$ is given by Eq. (1a). The Zeeman frequency shift induced by the static applied magnetic field $\overrightarrow{\mathcal{B}}$ is given by $\Delta \omega_{\text {Zeeman }}=\left(\gamma_{4}-\gamma_{3}\right)|\overrightarrow{\mathcal{B}}| m_{F}$, where the $\gamma_{F}$ are the gyromagnetic ratios of the hyperfine levels $F$.

In a monochromatic beam, all atoms will have the same time of flight $T$ between the pump and probe zones, leading to a cosinelike oscillatory dependence (16) on the microwave detuning. Averaging over the broad velocity distribution of the thermal beam used in the experiment will impose the typical Ramsey-type envelope (17) on that oscillatory structure. The velocity distribution $\rho(v)$ is not known a priori since it differs appreciably from a perfect Maxwell-Boltzmann distribution in our effusive beam, with the discrepancy arising from atomic collisions in the oven's nozzle and collimation regions [24]. 


\section{E. Fourier transform of the Ramsey spectrum}

The real and imaginary parts of the (inverse) Fourier transform of Eq. (17) are given by

$$
\begin{aligned}
A(t) & \equiv \operatorname{Re}\left\{\mathcal{F}^{-1}\left[S\left(\omega_{\mu \mathrm{w}}\right)\right]\right\} \\
& =\sqrt{\frac{\pi}{2}} \rho(t) \frac{L}{t^{2}} \cos \left[\left(\omega_{0}+\Delta \omega\right) t-\Delta \varphi\right],
\end{aligned}
$$

and

$$
\begin{aligned}
B(t) & \equiv \operatorname{Im}\left\{\mathcal{F}^{-1}\left[S\left(\omega_{\mu \mathrm{w}}\right)\right]\right\} \\
& =\sqrt{\frac{\pi}{2}} \rho(t) \frac{L}{t^{2}} \sin \left[\left(\omega_{0}+\Delta \omega\right) t-\Delta \varphi\right],
\end{aligned}
$$

where $\Delta \omega$ represents the sum of the Zeeman and Stark shifts in Ref. (17). These Fourier transforms allow the determination of the time-of-flight distribution,

$$
\rho(t)=\frac{t^{2}}{L} \sqrt{\frac{2}{\pi}} \sqrt{A^{2}(t)+B^{2}(t)},
$$

between the pump and probe zones (separated by $L$ ), and the phase

$$
\left(\omega_{0}+\Delta \omega\right) t-\Delta \varphi=\arctan \left[\frac{B(t)}{A(t)}\right],
$$

accumulated by the atoms on their flight from the pump to the probe zone.

\section{F. Effects of a static electric field on the Ramsey pattern}

Subjecting the atoms to an electric field during their flight between the pump and probe interactions changes the energies of the hyperfine sublevels both via the Stark interaction proper and via the Zeeman effect induced by the motional magnetic field experienced by the atoms moving through the transverse electric field. Both effects appear simultaneously and modify the Ramsey line shape of Eq. (17) in different ways, due to their different velocity dependencies.

The frequency shift $\Delta \omega_{\text {Stark }}$ that the $\Delta m_{F}=0$ coherence acquires in the free evolution zone $L$ due to the direct Stark interaction with an electric field of spatial distribution $\overrightarrow{\mathcal{E}}(z)$ is given by

$$
\begin{aligned}
\Delta \omega_{\text {Stark }} & =-\frac{1}{2} \alpha\left(m_{F}\right)\left\langle|\overrightarrow{\mathcal{E}}|^{2}\right\rangle_{L} \\
& =-\frac{1}{2} \alpha\left(m_{F}\right) \frac{1}{L} \int_{0}^{L}|\overrightarrow{\mathcal{E}}(z)|^{2} d z,
\end{aligned}
$$

where $\alpha\left(m_{F}\right)$ was introduced in Eq. (1b). The Stark shift proper will thus shift the entire Ramsey spectrum (fringes plus envelope) along the $\omega_{\mu \mathrm{w}}$ axis, as shown on the top of Fig. 2.

The electric field has a second effect on the Ramsey spectrum. In the reference frame of atoms moving at velocity $\vec{v}$ through the (transverse) electric field appears a motional magnetic field that is given, to first order in $v / c$, by

$$
\overrightarrow{\mathcal{B}}_{\mathrm{mot}}=\frac{\vec{v}}{c^{2}} \times \overrightarrow{\mathcal{E}}
$$

For a typical mean atomic velocity $\bar{v}$ of $\sim 250 \mathrm{~m} / \mathrm{s}$ and an electric field strength $|\overrightarrow{\mathcal{E}}|$ of $\sim 20 \mathrm{kV} / \mathrm{cm}$, the motional field is $\left|\overrightarrow{\mathcal{B}}_{\text {mot }}\right| \sim 5 \mathrm{nT}$ for $\overrightarrow{\mathcal{E}} \perp \vec{v}$. Atoms with a $\Delta m_{F}=0$ coherence

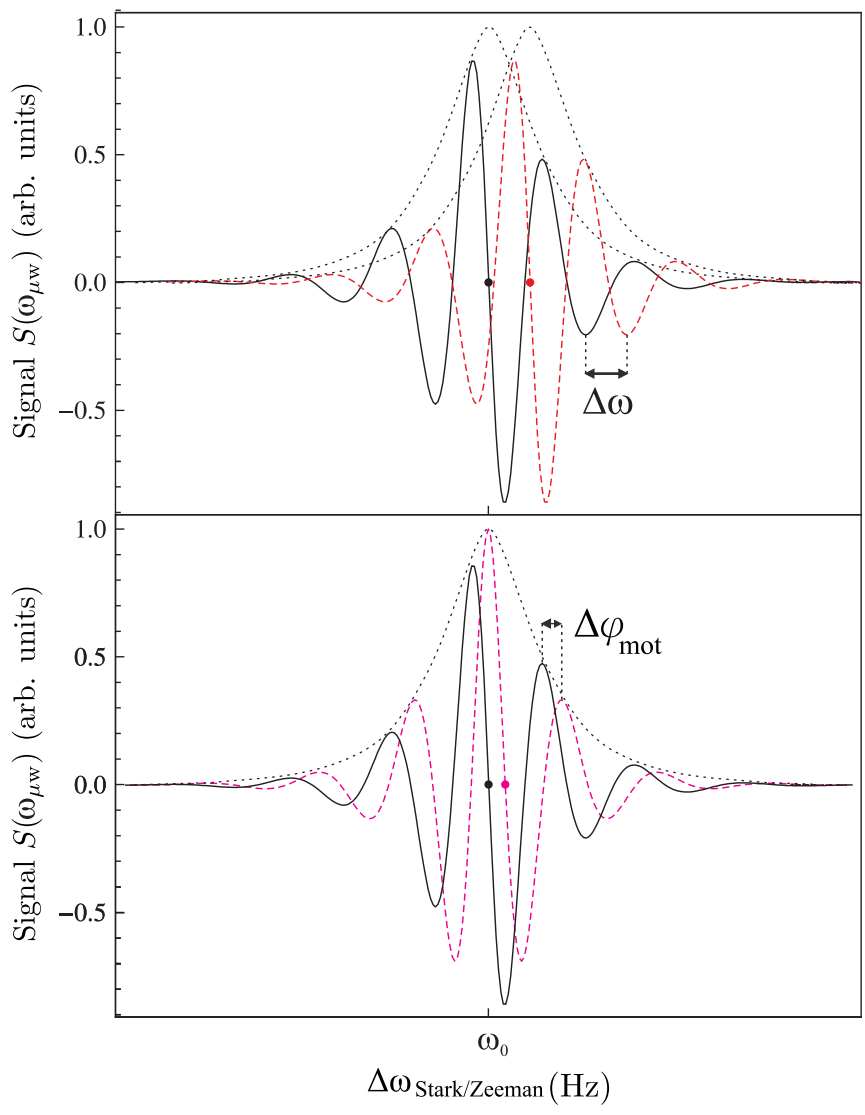

FIG. 2. (Color online) Expected unperturbed dispersive Ramsey line shape for $\Delta \varphi_{\text {path }}=\pi / 2$ (black line) and its change (red dashed line) by a frequency shift $\Delta \omega=\Delta \omega_{\text {Stark }}$ (or $\Delta \omega_{\text {Zeeman }}$ ) (top) and the motional phase shift $\Delta \varphi_{\text {mot }}$ (bottom). The frequency shift displaces both the fringes and their envelope, while the phase shift displaces the fringes under the envelope. Both effects, therefore, displace the zero crossings (marked by dots).

will thus acquire, via the linear Zeeman effect induced by $\overrightarrow{\mathcal{B}}_{\text {mot }}$, a phase shift

$$
\begin{aligned}
\Delta \varphi_{\mathrm{mot}} & =\frac{\gamma_{4}-\gamma_{3}}{c^{2}} m_{F} \sin (\xi) v \int_{0}^{T}|\overrightarrow{\mathcal{E}}(t)| d t \\
& =\frac{\gamma_{4}-\gamma_{3}}{c^{2}} m_{F} \sin (\xi) \int_{0}^{L}|\overrightarrow{\mathcal{E}}(z)| d z,
\end{aligned}
$$

where $T$ is the pump-probe flight time of an atom with velocity $v$, and $\xi$ is the angle between $\vec{v}$ and $\overrightarrow{\mathcal{E}}$. In contrast to the frequency shift induced by the Stark interaction of interest, the motional field effect is a topological (velocity-independent) phase shift that manifests itself as a displacement of the Ramsey fringes under their otherwise fixed envelope, as shown in the bottom graph of Fig. 2. We note that both effects displace the zero crossings of the Ramsey fringes.

\section{EXPERIMENTAL APPARATUS}

The main elements of the apparatus have been presented in Refs. [21-23] and are shown in Fig. 3. We have used two methods for measuring the differential Stark shift of the Cs clock transition. Some elements of the experimental method 


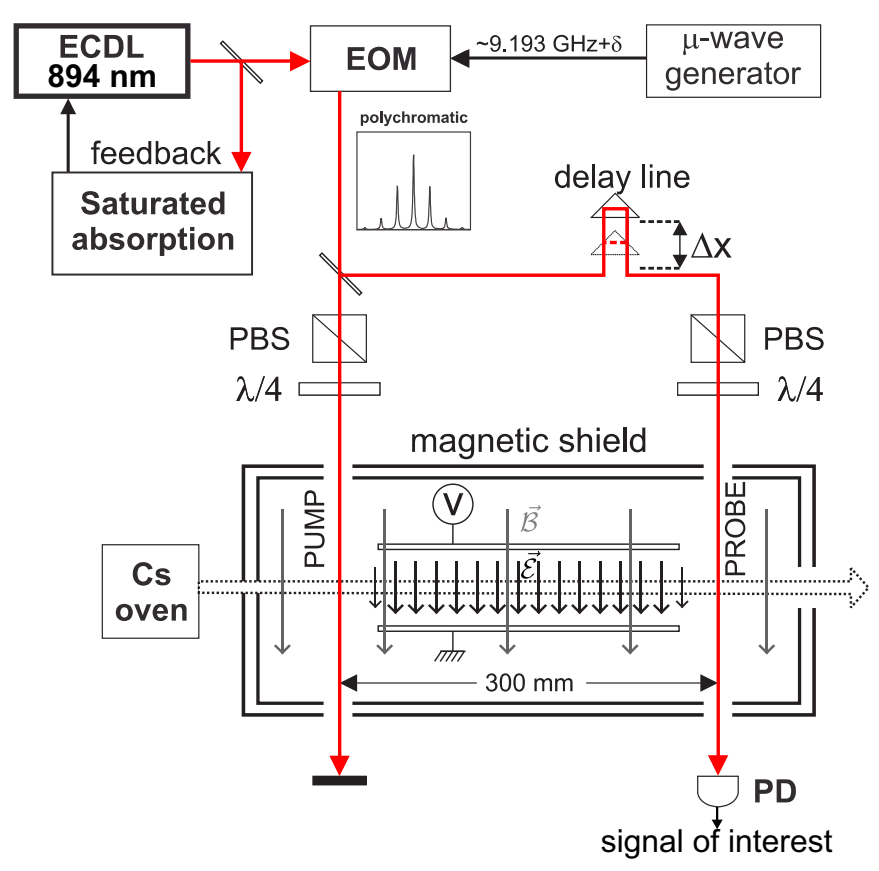

FIG. 3. (Color online) General experimental configuration of the Ramsey interrogation scheme. Details are given in the text.

used to lock the microwave frequency to the Ramsey fringe center were presented in Ref. [25] and a preliminary data analysis using the Fourier-transform method was reported in Refs. [22,23]. Here we present the final analysis and results based on data obtained with the fringe-tracking and Fouriertransform methods, respectively.

\section{A. Atomic beam}

A thermal Cs atomic beam is produced by an effusive reflux oven inspired by the design described in Ref. [26]. Additional collimating elements are placed along the atomic beam trajectory to restrict the beam's cross section (horizontal and vertical divergence angles of \pm 4.5 and $\pm 8.5 \mathrm{mrad}$, respectively) to a rectangular profile of $9 \mathrm{~mm}$ along $\hat{x}$ and $4 \mathrm{~mm}$ along $\hat{y}$ in the Ramsey interrogation zone. The atomic densities in the pump and probe interaction regions are estimated to be $\sim 50 \times 10^{6} / \mathrm{cm}^{3}$ and $\sim 16 \times 10^{6} / \mathrm{cm}^{3}$, respectively. The pump, probe, and electric-field regions are enclosed in a vacuum chamber with typical pressure of $5 \times 10^{-7}$ mbar surrounded by a cylindrical two-layer $\mu$-metal shield. A nominally homogeneous transverse magnetic field of 3.57(1) $\mu \mathrm{T}$ is applied to all regions of interest. The laser beams enter and leave the vacuum chamber through 10-mm-diameter windows.

\section{B. Electric-field generation and calibration}

A complete view of the field generating capacitor and its dielectric support structure is shown in Fig. 4. The electrodes are made of two rectangular $(50 \times 260 \times 4 \mathrm{~mm})$ float glass plates with a conductive coating. The plate spacing is defined by 10 optical flats with a thickness of $6.065(1) \mathrm{mm}$, inserted in an insulating polycarbonate holder. Two grounded metal plates at each end of the capacitor are used to collimate the Cs

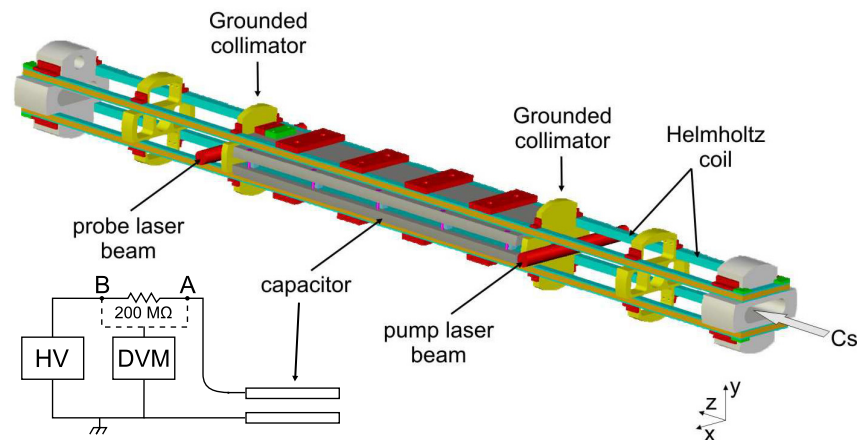

FIG. 4. (Color online) View of the electrode support structure. Grounded metallic collimator plates placed upstream and downstream of the capacitor prevent the fringe fields from perturbing the pump and probe interaction zones. Long rectangular Helmholtz coils produce a homogeneous dc magnetic field parallel to the electric field over the entire Ramsey interaction region. Inset: the electrical connection scheme.

beam and to prevent the electric fringe fields from perturbing the two optical interaction regions.

High voltage, provided by a Heinzinger PNC 60000-1ump power supply capable of delivering up to $60 \mathrm{kV}$ with a stability of $10^{-4}$ over eight hours, is applied to one electrode while the other electrode is grounded. The maximum voltage used in the experiment was $10 \mathrm{kV}$ to avoid sparking inside the vacuum chamber. The electrode voltage is measured by a digital voltmeter (DVM) with a resolution of $10^{-4}$; the device was calibrated just before the final measurements at the Swiss metrology institute METAS. A $200 \mathrm{M} \Omega$ protection resistor between the generator and the high-voltage electrode is used to limit the current, and hence the destructive power, of any sparks generated during breakdown. For the Fourier-transform measurements described below, the voltage drop over the fieldproducing capacitor was measured directly (DVM measuring at the capacitor plate, point "A"), while for the fringe-tracking experiments, we measured the voltage at the power supply (DVM connected at point "B"), leading-because of leakage currents through the resistor-to an increased uncertainty of the electric field.

In order to determine the average electric field applied to the atoms from measurement of the voltage applied to the electrodes, the electric field's spatial distribution was modeled by Dr. Z. Andjelić [ABB Corporate Research in Baden $(\mathrm{CH})$ ], using the code POLOPT [27] that is based on the advanced boundary integral method in three dimensions described in Ref. [28]. The modeling relied on the accurate representation of the mechanical structures supporting the capacitor plates, including all surfaces, conductors, and insulators in the region between the two grounded collimation slits. The model calculates the electric field throughout the volume for a potential of $1 \mathrm{~V}$ applied to one electrode, with the other held at ground, and assumes that the field increases linearly with the applied voltage. The obtained electric-field distribution for one atomic path is shown in Fig. 5 as well as the three-dimensional (3D) model used for the calculation.

The three vector components of $\overrightarrow{\mathcal{E}}$ are calculated every $1.75,1.5$, and $2 \mathrm{~mm}$ along the $x, y$, and $z$ axis, respectively. 


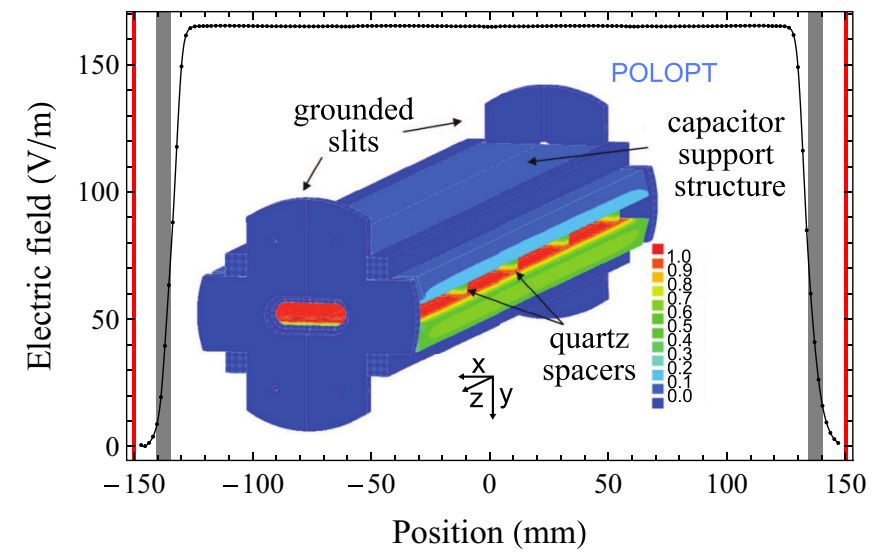

FIG. 5. (Color online) Example of computed electric-field distribution along the central atomic beam path in the capacitor. The red lines show the positions of the pump and probe laser beam, while the gray bands represent the grounded slits. The field asymmetry in the spacing between the lasers and grounded slits on the two sides of the capacitor is due to the different geometry and size of the input and output slits. In the center, the picture shows the 3D model used for the simulation, with the color scale representing the voltage from 0 to $1 \mathrm{~V}$.

Since the modeling produces the electric field throughout all space between the electrodes, the mean-field values and their standard deviations are evaluated for several possible atomic trajectories. Along the $y$ axis, the trajectories that contribute to the signal are limited by the probe laser beam's extension of $\pm 1.2 \mathrm{~mm}$. Figure 6 represents an "atom's-eye" view of the apparatus and shows the positions of the trajectories chosen for averaging.

In order to relate the frequency shift $\Delta \omega_{\text {Stark }}$ and the motional phase shift $\Delta \varphi_{\text {mot }}$ to the field integrals in Eqs. (26b) and (28b), respectively, one has to know the pump-probe

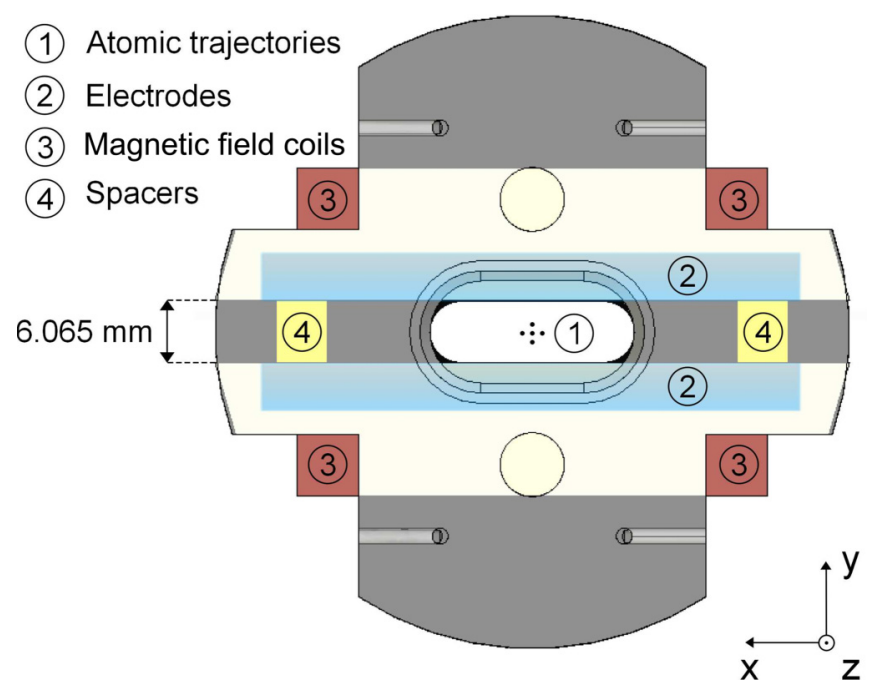

FIG. 6. (Color online) Inbound-atom view of the second collimator plate. This corresponds to a section view of the electrode support of Fig. 4 at the "capacitor" label position. The five dots at the center show the atom trajectory positions considered in the electric-field modeling.
TABLE I. Numerical values for the two electric-field integrals defined by Eqs. (29) obtained by field modeling, with a pump-probe separation $L=301.8(7) \mathrm{mm}$ and a potential difference of $1 \mathrm{~V}$. The uncertainty on the individual path calibration constants is dominated by the uncertainty on $L$; however, the uncertainty on the average is the standard deviation of the values.

\begin{tabular}{lcr}
\hline \hline Trajectory $(x, y)$ & $I_{1}(\mathrm{~V})$ & $I_{2}(\mathrm{~V} / \mathrm{cm})^{2}$ \\
\hline$(0,0)$ & 44.4208 & $2.393(6)$ \\
$(0,+1.5 \mathrm{~mm})$ & 44.9346 & $2.434(6)$ \\
$(0,-1.5 \mathrm{~mm})$ & 44.2066 & $2.382(6)$ \\
$( \pm 1.75 \mathrm{~mm}, 0)$ & 44.4154 & $2.392(6)$ \\
Average & $44.48(12)$ & $2.399(9)$ \\
\hline \hline
\end{tabular}

separation $L$. This distance was measured directly on the apparatus by determining the separation of the centers of the pump and probe beams' intensity distributions. In order to account for a possible nonparallelism of the beams, the separations measured near the entrance and exit windows of the vacuum chamber were averaged, yielding $L=301.8(7) \mathrm{mm}$.

The $L$-averaged electric-field integrals, defined as

$$
\begin{aligned}
& I_{1}=\int_{0}^{L}|\overrightarrow{\mathcal{E}}(z)| d z \equiv \frac{L}{d_{\mathrm{eff}}}(1 \mathrm{~V}), \\
& I_{2}=\frac{1}{L} \int_{0}^{L}|\overrightarrow{\mathcal{E}}(z)|^{2} d z \equiv \frac{1 \mathrm{~V}^{2}}{\left(d^{2}\right)_{\mathrm{eff}}},
\end{aligned}
$$

for the five paths are given in Table I, along with the final average value.

Since the modeling calculations were done for a voltage of $1 \mathrm{~V}$, the integrals can be parametrized in terms of effective plate spacings $d_{\text {eff }}$ and $\left(d^{2}\right)_{\text {eff }}$, defined by Eqs. (29), that represent the effect of the capacitor's finite size. The numerical values of the effective spacings from the modeling calculation are compared to the geometrical electrode spacing in Table II.

\section{The bichromatic laser fields}

The (single) laser used for the pump-probe experiments is a $40 \mathrm{~mW}$ extended cavity diode laser emitting monochromatic radiation near the Cs $D_{1}$ transitions ( $~ 894.6 \mathrm{~nm}$ ) with a spectral linewidth below $1 \mathrm{MHz}$. The laser wavelength is actively stabilized to the $F=3 \rightarrow F^{\prime}=3$ hyperfine component of

TABLE II. Comparison of electrode spacings. Upper two values: Effective spacings from the modeled average value of the electric field and its square as defined by Eqs. (29) with values from Table I. Bottom: Geometrical electrode spacing determined by spacers. The difference between geometrical and effective separations is dominated by the ratio of $L=301.8 \mathrm{~mm}$, the pump-probe distance, to the physical capacitor length of $260.0 \mathrm{~mm}(260.0 / 301.8 \mathrm{~mm}=0.86$, $d_{\text {geom }} / d_{\text {eff }}=0.89$ ), with the remaining difference due to the structure of the fringe fields.

\begin{tabular}{lc}
$d_{\text {eff }}=L / I_{1}$ & $6.79(2) \mathrm{mm}$ \\
$\left(d^{2}\right)_{\text {eff }}=1 / I_{2}$ & $41.69(16) \mathrm{mm}^{2}$ \\
$\sqrt{\left(d^{2}\right)_{\mathrm{eff}}}=1 / \sqrt{I_{2}}$ & $6.457(12) \mathrm{mm}$ \\
$d_{\text {geom }}$ & $6.065(1) \mathrm{mm}$ \\
\hline \hline
\end{tabular}


the $D_{1}$ line using Doppler-free spectroscopy in an auxiliary Cs vapor cell.

The phase-coherent bichromatic light field needed for the CPT pump-probe scheme is provided by a polarization maintaining fiber coupled lithium niobate electro-optic phase modulator (EOM, Photline, model NIR-MPX800-LN08). The EOM is driven by a frequency-tunable microwave source (Rohde \& Schwarz, model SMP 02 signal generator) capable of delivering frequencies from $10 \mathrm{MHz}$ to $20 \mathrm{GHz}$ with a resolution of $0.1 \mathrm{~Hz}$, but here used to create $v_{\mu \mathrm{w}}$ close to the Cs hyperfine frequency of $9.2 \mathrm{GHz}$. The microwave generator is referenced to the $10 \mathrm{MHz}$ signal from an atomic clock (Temex, model PFRS Rb clock) for better stability and accuracy. The EOM creates sidebands at positive and negative integer multiples of $v_{\mu \mathrm{w}}$ around the laser carrier frequency. The carrier and first sideband form the two components of the phase-coherent bichromatic field. The modulation index is chosen to yield identical Rabi frequencies of the two transitions $\left(6 S_{1 / 2}, F=3 \rightarrow 6 P_{1 / 2}, F^{\prime}=3\right.$ and $6 S_{1 / 2}, F=$ $4 \rightarrow 6 P_{1 / 2}, F^{\prime}=3$ ), whose oscillator strengths are in the ratio $1: 3$, and that are driven coherently by the bichromatic field.

A $\sim 500 \mu \mathrm{W}$ pump beam and a $\sim 10 \mu \mathrm{W}$ probe beam are derived from the modulated beam using a beam splitter, as schematized in Fig. 3. The probe beam path length and hence the spatial phase factor given by Eq. (16) are varied by a delay line formed by two orthogonal mirrors on a micrometer-controlled linear translation stage. Each change of the optical path-length difference $\Delta L$ by one microwave wavelength $\left(\lambda_{\mu \mathrm{w}} \cong 32.6 \mathrm{~mm}\right.$ for $\left.\nu_{\mu \mathrm{w}}=9.192 \mathrm{GHz}\right)$ changes the phase of the Ramsey pattern by $2 \pi$. Phase adjustments were made with no electric field applied to the atoms.

\section{Heterodyne detection}

Two distinct heterodyne detection methods, shown in Fig. 7 , were used to measure the shift of the central Ramsey fringe's frequency as a function of the applied electric field applied to the atoms.

The first method consists in recording complete Ramsey spectra by monitoring the transmitted probe laser power while scanning the microwave frequency $\omega_{\mu \mathrm{w}}$ over a given $\left|3, m_{F}\right\rangle \rightarrow\left|4, m_{F}\right\rangle$ transition. In these experiments, the pump beam components were $\sigma^{+}$polarized and the field configuration was $\overrightarrow{\mathcal{E}} \perp \overrightarrow{\mathcal{B}}$, corresponding to $\theta=\pi / 2$ in Eq. (1a). In the probe region, we measured the circular dichroism of the beam by switching the probe beam polarization between $\sigma^{+}$ and $\sigma^{-}$states using a photoelastic modulator (PEM, Hinds Instruments, model I/FS50). The transimpedance-amplified (400 kHz bandwidth) photodiode signal was analyzed by a lock-in amplifier referenced to the modulation frequency $(50 \mathrm{kHz})$ of the PEM.

In the second method, the microwave frequency was actively locked to the central Ramsey fringe's zero crossing and the electric-field-induced frequency shift of the resonance frequency was inferred from the feedback signal. For these experiments, the pump-probe path-length difference $\Delta x$ was set to a multiple of $\lambda_{\mu \mathrm{w}}$, thus yielding cosinelike Ramsey fringes (the absorptive counterpart of the spectra shown in Fig. 2). The frequency of the microwave oscillator was modulated by $23 \mathrm{kHz}$ with a frequency of $525 \mathrm{~Hz}$ and

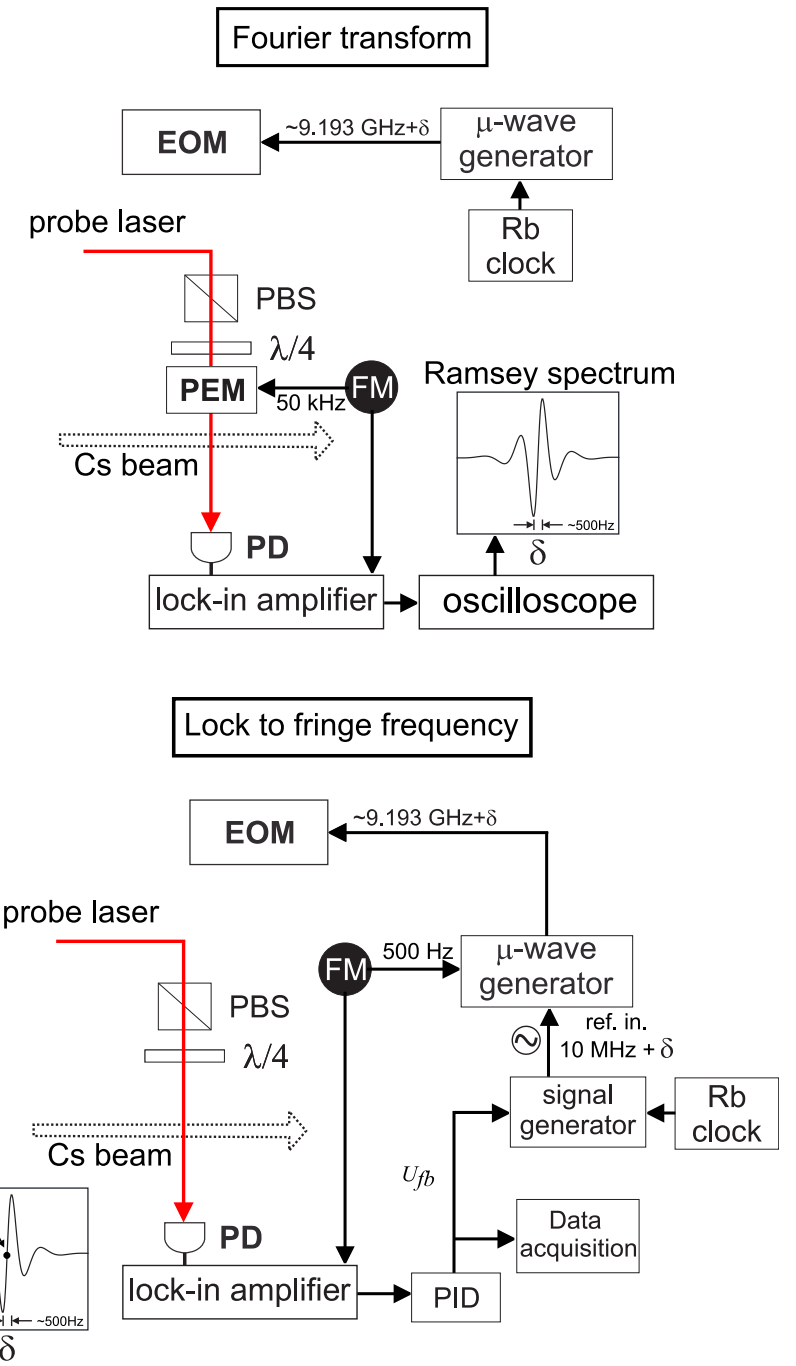

FIG. 7. (Color online) Experimental schemes of the two heterodyne detection methods deployed here. Top: Heterodyne detection used to record Ramsey spectra for Fourier-transform analysis. The polarization of the probe beam is modulated between $\sigma^{+}$and $\sigma^{-}$with a photoelastic modulator (PEM) at $50 \mathrm{kHz}$. Bottom: The slope of the dispersive Ramsey fringe's zero crossing is used as a discriminant to lock the microwave frequency to the fringe center. The heterodyne measurement is done by modulating the microwave generator frequency, and the error signal, after PID amplification, is sent to the frequency modulation input of the $10 \mathrm{MHz}$ reference of the microwave generator.

the probe detector's photocurrent demodulated by a lock-in amplifier locked to that modulation frequency. Scanning the microwave frequency $\omega_{\mu \mathrm{w}}$ then yields a dispersive fringe pattern (the derivative of the absorptive cosine pattern) similar to the one shown in Fig. 2. The near-resonance linear zero crossing of this signal is used as a discriminator signal to form, after a proportionnal-integral-derivative (PID) controller amplification, a feedback signal $U_{\mathrm{fb}}$ controlling the frequency of the microwave oscillator. This control was achieved in the following way: The $\mathrm{Rb}$ clock references a function generator (Agilent, model 33220A), which generates the $10 \mathrm{MHz}$ reference signal for the microwave generator. In order to lock the microwave frequency to the atomic signal, the 
$10 \mathrm{MHz}$ generated by the function generator is controlled by applying $U_{\mathrm{fb}}$ to the generator's FM control input. The feedback signal $U_{\mathrm{fb}}$ in the locked mode is recorded on a digital oscilloscope for $\sim 600 \mathrm{~s}$ and its average value is determined as a function of the applied electrode voltage $U$. The calibration constant converting the feedback voltage into frequency units is used to express the displacement of the Ramsey fringe center in $\mathrm{Hz}$. The pump and probe laser beams were $\sigma^{+}$polarized for experiments with $\overrightarrow{\mathcal{E}} \perp \overrightarrow{\mathcal{B}}$ and linearly polarized for $\overrightarrow{\mathcal{E}} \| \overrightarrow{\mathcal{B}}$.

\section{MEASUREMENTS}

\section{A. CPT-Ramsey spectra with $\mathcal{E}=\mathbf{0}$}

Figure 8 shows a large range scan of the microwave frequency near the $\left|6 S_{1 / 2}, F=3\right\rangle \rightarrow\left|6 S_{1 / 2}, F=4\right\rangle$ Raman transition. The width of $\sim 9 \mathrm{MHz}$ reflects the efficiency of the Raman process that is limited by the (power-broadened) width of the excited $6 P_{1 / 2}$ state. The ( $\sim 400-\mathrm{kHz}$-wide) dip in the top spectrum is a strongly power-broadened CPT resonance that occurs in the pump region. Here the pump beam acts both as pump and probe, similar to microwave CPT spectroscopy in vapor cells.

The middle graph of Fig. 8 is a zoom of the bottom of that CPT dip. It reveals six individual $\Delta m_{F}=0$ Ramsey resonances that are split by the $3.6 \mu \mathrm{T}$ magnetic field applied to the beam. We note that each resonance is superposed on a curved background that varies from resonance to resonance. Finally, the lower graph of Fig. 8 shows the Ramsey pattern of the $\left|6 S_{1 / 2}, F=3, m_{F}=0\right\rangle \rightarrow\left|6 S_{1 / 2}, F=4, m_{F}=0\right\rangle$ clock transition (after background removal by fitting), whose Stark shift is the object of the measurement reported below. All graphs represent the circular dichroism recorded in the probe zone by the method described in Sec. III D yielding dispersive fringes since $\Delta \varphi_{\text {path }}=\pi / 2$ for this measurement.

\section{B. $\mathcal{E}$-field-induced frequency and phase shift}

As discussed in Sec. II F, a static electric field will change both the frequency and the phase of the Ramsey fringes. The polarizability of interest, defined by Eqs. (1a) and (1b), is inferred from the electric-field-induced frequency shift of the Ramsey fringe pattern (17) via

$$
\alpha\left(m_{F}\right)=-2 \frac{\Delta v_{\text {Stark }}\left(m_{F}\right)}{\left\langle|\overrightarrow{\mathcal{E}}|^{2}\right\rangle_{L}}=-2 \frac{\Delta v_{\text {Stark }}\left(m_{F}\right)}{U^{2}}\left(d^{2}\right)_{\mathrm{eff}}
$$

where $\Delta v_{\text {Stark }}$ is obtained from the difference of the central fringe's center frequency when measured with and without applied electric field. The phase shift of the fringes due to the motional magnetic field is used to test the field modeling predictions. The following sections describe the results obtained by the two methods introduced in Sec. III D for determining that center frequency.

\section{Stark shifts from Fourier-transform analysis}

In order to apply the Fourier transformation described in Sec. IIE, we have recorded Ramsey spectra, such as the one shown in the bottom graph of Fig. 8, for different voltage differences $U$ applied to the electric-field plates. Spectra were taken by scanning the microwave frequency over a typical
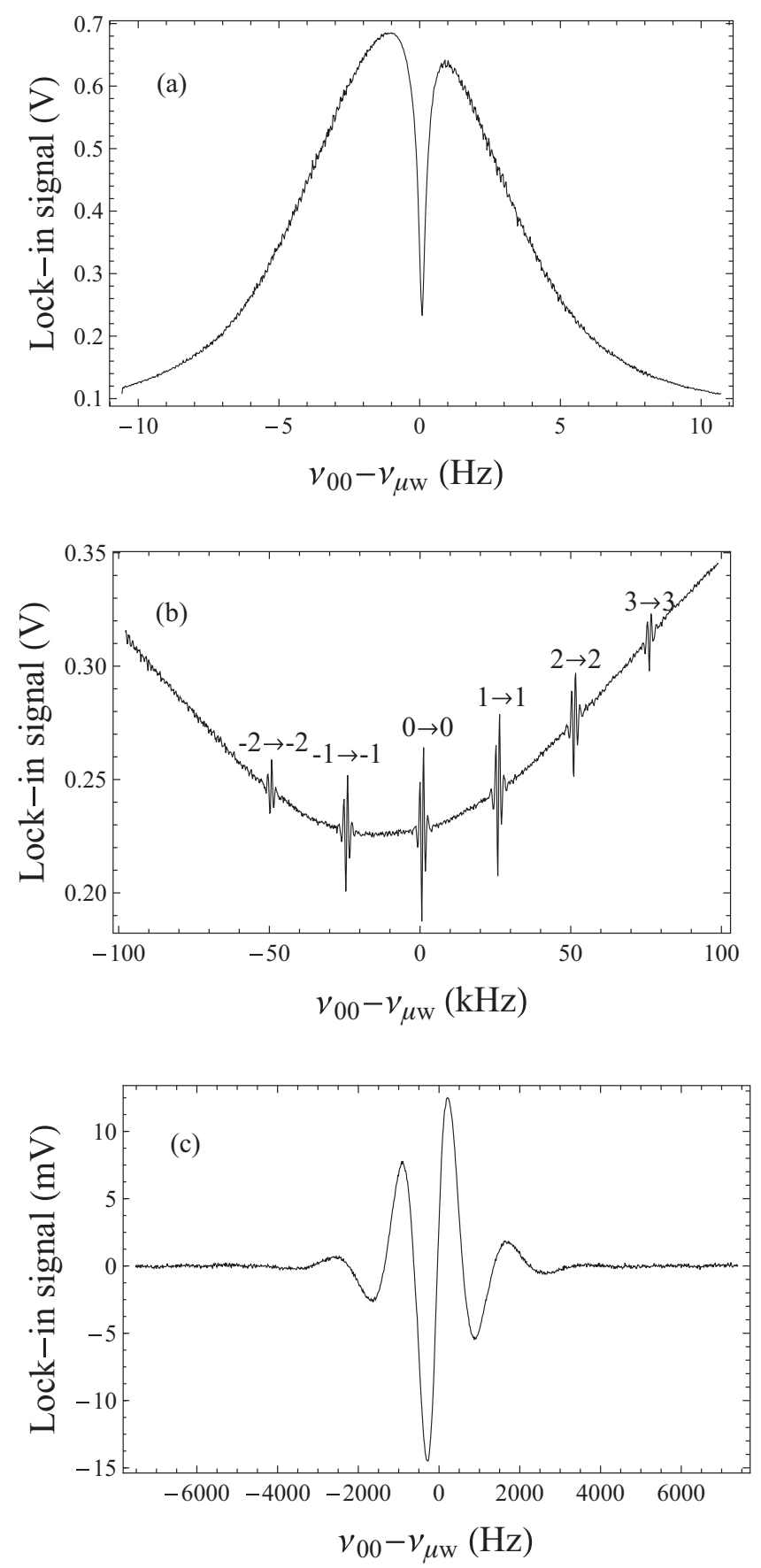

FIG. 8. (a) Probe beam spectrum of the unresolved $\left|6 S_{1 / 2}, F=4, m_{F}\right\rangle \rightarrow\left|6 P_{1 / 2}, F=3, m_{F}\right\rangle$ transitions as a function of the Raman frequency, i.e., the microwave frequency producing the laser sidebands. The central dip represents the coherent-populationtrapping effect. (b) Zoom of the bottom of the dip revealing the resolved $m_{F} \rightarrow m_{F}^{\prime}=m_{F}$ transitions split by the $3.57(1) \mu \mathrm{T}$ magnetic field, together with quadratic background. (c) Closeup of the $m_{F}=0 \rightarrow m_{F}^{\prime}=0$ clock transition after background subtraction. In all graphs, the microwave frequency $v_{\mu \mathrm{w}}$ is measured with respect to the clock frequency $v_{00}=9.192631770 \mathrm{GHz}$.

span of $15 \mathrm{kHz}$, in steps of $15 \mathrm{~Hz}$ with $20 \mathrm{~ms}$ dwell time at each frequency value. The output of the lock-in amplifier measuring the circular dichroism seen by the probe beam (cf. Sec. III D) was recorded on a digital oscilloscope, and typically 


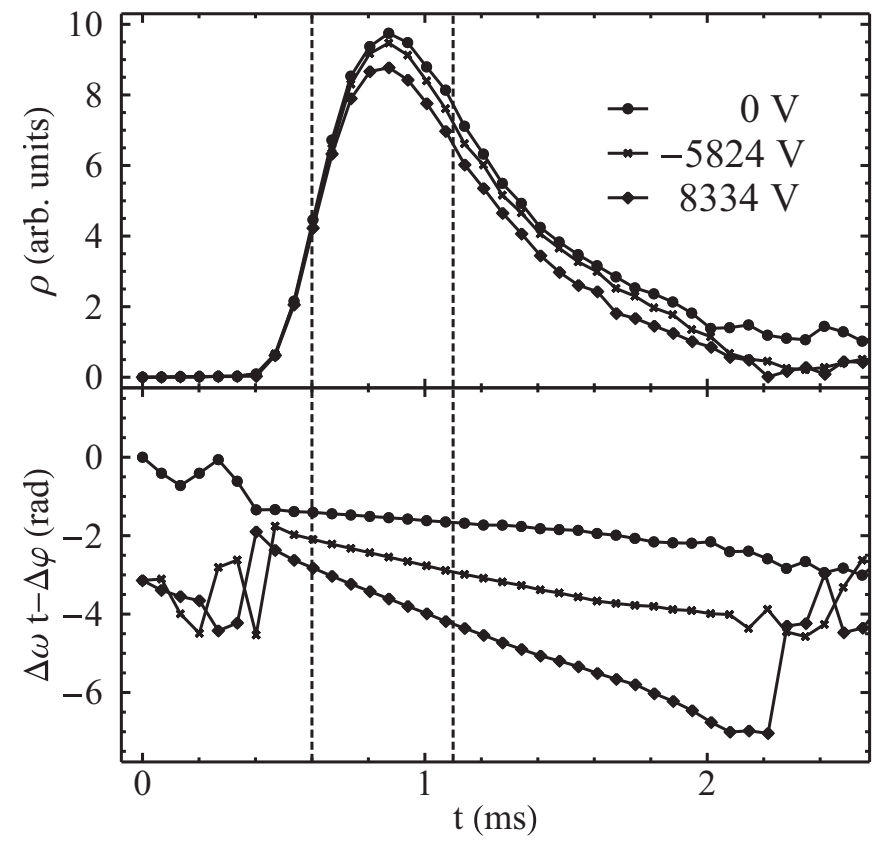

FIG. 9. Time-of-flight distribution $\rho(t)$ (top) and phase $\Delta \omega t-$ $\Delta \varphi$ (bottom), obtained by Fourier transformation of the Ramsey signals under different electric-field conditions. Data between the vertical dashed lines were used to infer the frequency $\Delta \omega(U)$ and phase $\Delta \varphi(U)$ shifts of interest.

24 complete scans over each resonance were averaged to yield one Ramsey spectrum as a function of the microwave frequency.

The curved background (seen in the middle graph of Fig. 8) was removed by fitting the left and right side of each fringe pattern with a second-order polynomial, and subtracting the fit result from the whole spectrum, yielding the signal $S\left(\omega_{\mu \mathrm{w}}, U\right)$. Following each two measurements with applied voltage, a reference spectrum $S\left(\omega_{\mu \mathrm{w}}, U=0\right)$ with no applied voltage was recorded.

We next performed numerical Fourier transforms (FT) of the spectra $S\left(\omega_{\mu \mathrm{w}}, U\right)$ and $S\left(\omega_{\mu \mathrm{w}}, 0\right)$ recorded with and without applied voltage to yield the time-of-flight distribution $\rho(t)$ and the phase $\Delta \omega t-\Delta \varphi$. A typical result is shown in Fig. 9. The data show that the phase obeys a linear time dependence only for a restricted range of time of flights. The signal becomes very noisy for very fast and very slow atoms, whose density $\rho(t)$ is small. While all time-of-flight distributions are mutually consistent below $t \sim 1.5 \mathrm{~ms}$, the distribution shows a background for $t>1.5 \mathrm{~ms}$ that increases with the electricfield intensity. This systematic dependence on the applied voltage can be seen in Fig. 10, where we have superposed (peak-normalized) time-of-flight distributions recorded with different applied fields for the $m_{F}=0 \rightarrow m_{F^{\prime}}=0$ transition. Although several interpretations of this effect (including field-dependent forces on the atoms in the field entrance and exit regions due to the electric-field gradients) were attempted, we could find no model explaining this feature in a quantitative way. The hyperfine coherence of slow atoms thus seems to acquire a field-dependent phase shift of unexplained origin.

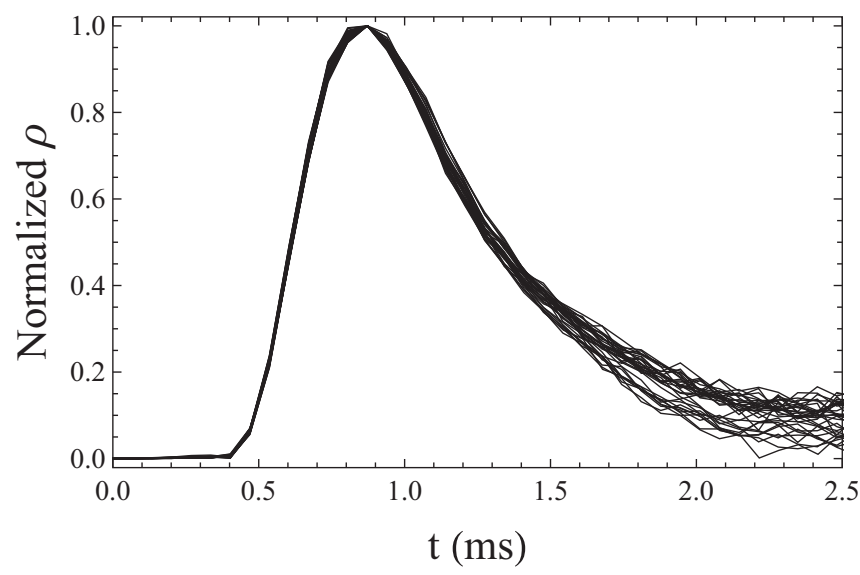

FIG. 10. Normalized time-of-flight distributions for all Ramsey spectra of the $m_{F}=0 \rightarrow m_{F^{\prime}}=0$ transitions recorded with different applied voltages. For times above $\sim 1.6 \mathrm{~ms}$, the electric field affects the relative atom density.

Because of the above, we have restricted the time range used to fit the data to $0.6 \leqslant t \leqslant 1.1 \mathrm{~ms}$, an interval where $\rho(t)$ has significant density and where the phase is well represented by Eq. (25), independent of the applied field. Our error analysis is based on variations of those fit limits. The phase data in the chosen time interval were fitted by a linear time dependence, and the electric-field-induced frequency and phase shifts obtained from the fitted slope and intercept according to

$\Delta v_{\text {Stark }}=\Delta v(U)-\Delta v(0)$ and $\Delta \varphi_{\text {mot }}=\Delta \varphi(U)-\Delta \varphi(0)$.

The Stark shifts $\Delta v_{\text {Stark }}$ of the clock frequency extracted in this way are presented in Fig. 11 together with their fit by a second-order polynomial of the form $\Delta v_{\text {Stark }}=c_{0}+c_{1} U+c_{2} U^{2}$. The fitted coefficients $c_{0}=$ $0.3(9) \mathrm{Hz}$ and $c_{1}=-180(110) \mathrm{mHz} / \mathrm{kV}$ are consistent with zero, as expected.

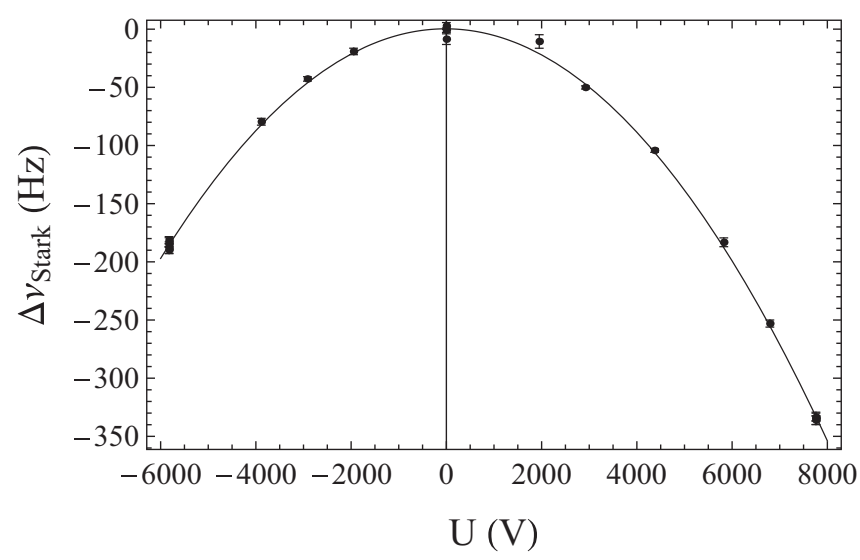

FIG. 11. Quadratic Stark shift of the $6 S_{1 / 2}, F=3, m_{F}=0 \rightarrow$ $6 S_{1 / 2}, F=4, m_{F}=0$ clock transition frequency determined by the Fourier analysis method. The solid line is a fit with a second-order polynomial. 
The scalar polarizability $\alpha_{0}^{(3)}(F=4)$ is related to the parameter $c_{2}$ by

$$
\alpha_{0}^{(3)}=-\frac{7}{8} c_{2}\left(d^{2}\right)_{\mathrm{eff}}-\frac{1}{4} \alpha_{2}^{(3)} .
$$

Using the fitted value $c_{2}=-5.51(3)_{\text {stat }} \mathrm{Hz} / \mathrm{kV}^{2}$ and the literature-based $[19,20,29]$ weighted average $-3.51(16) \times$ $10^{-2} \mathrm{~Hz} /(\mathrm{kV} / \mathrm{cm})^{2}$ for the tensor polarizability $\alpha_{2}^{(3)}(F=4)$, we find

$$
\begin{aligned}
\alpha_{0}^{(3)} & =2.020(10)_{\text {stat }}(9)_{\text {syst }} \mathrm{Hz} /(\mathrm{kV} / \mathrm{cm})^{2} \\
& =2.020(13) \mathrm{Hz} /(\mathrm{kV} / \mathrm{cm})^{2},
\end{aligned}
$$

and

$$
\begin{aligned}
k_{s} & =-2.308(11)_{\text {stat }}(10)_{\text {syst }} \mathrm{Hz} /(\mathrm{kV} / \mathrm{cm})^{2} \\
& =-2.308(15) \mathrm{Hz} /(\mathrm{kV} / \mathrm{cm})^{2},
\end{aligned}
$$

where we have added the statistical and systematic uncertainties in Eqs. (33a) and (34a) quadratically to yield the global errors in Eqs. (33b) and (34b), respectively.

The systematic uncertainty is dominated by the $5 \times 10^{-3}$ precision of field calibration constant $\left(d^{2}\right)_{\text {eff }}$, to which adds an uncertainty of the pump-probe separation $L$. The distance between the centers of the pump and probe beams' intensity distributions was measured to be $L=301.1(1) \mathrm{mm}$. To correct for the fact that optical pumping occurs with a higher probability in the upstream part of the atomic laser beams' intersection volume, $L$ was lengthened by $\delta L$, taken as half of the $1 / e^{2}$ width of the pump laser beam, and the uncertainty on $L$ was increased accordingly. We therefore assign the value of $L+\delta L=301.8$ (7) $\mathrm{mm}$ to the pump-probe separation. When added quadratically to the uncertainty of $\left(d^{2}\right)_{\text {eff }}$, the $2 \times 10^{-3}$ effect due to $\delta L$ leads to a systematic error of $\sim 0.6 \%$.

Following Eq. (1a), the relative orientation $\theta$ of the (nominally orthogonal) electric and magnetic fields affects the contribution from $\alpha_{2}^{(3)}$ to $\alpha(0)$. A mismatch of $\Delta \theta=5^{\circ}$ from perfect orthogonality yields a relative change of only $10^{-4}$ in the total Stark shift. By construction, the orthogonality of the two fields is obeyed at the $\pm 0.5^{\circ}$ level, thus giving a negligible contribution the systematic error budget.

We have also measured the Stark shifts of the six $m_{F} \neq 0$ transitions. Their Fourier analysis revealed some $m_{F^{-}}$ dependent systematic phase perturbations that did not allow for an improved determination of the clock transition's Stark shift. However, when extrapolated to $t \rightarrow 0$, the values of the seven phase plots (equivalent to the one shown for $m_{F}=0$ in the bottom graph of Fig. 9) allowed the extraction of the phase $\Delta \varphi$ in Eq. (25). As discussed in Sec. II F, one expects a linear electric-field-dependent contribution $\Delta \varphi_{\text {mot }} \propto \mathcal{E} \propto U$ due to the motional Zeeman effect. Being a Zeeman shift, one further expects $\Delta \varphi_{\text {mot }}$ to have a linear dependence on $m_{F}$. Figure 12 illustrates the anticipated linear dependence of $\Delta \varphi_{\text {mot }}$ on $U$ and $m_{F}$ for all seven $\Delta m_{F}=0$ transitions. The increasing uncertainty with $m_{F}$ in Fig. 12 is due to the systematic perturbation mentioned above. The average slope of the fitted line in the figure is 20(1) $\mu \mathrm{rad} \mathrm{V}^{-1} m_{F}^{-1}$, while Eq. (28b), together with the field integral of Eq. (29a) and the modeled numerical value of Table II, yields 22(1) $\mu \mathrm{rad} \mathrm{V}^{-1} m_{F}^{-1}$, thus giving confidence in the field modeling calculations.

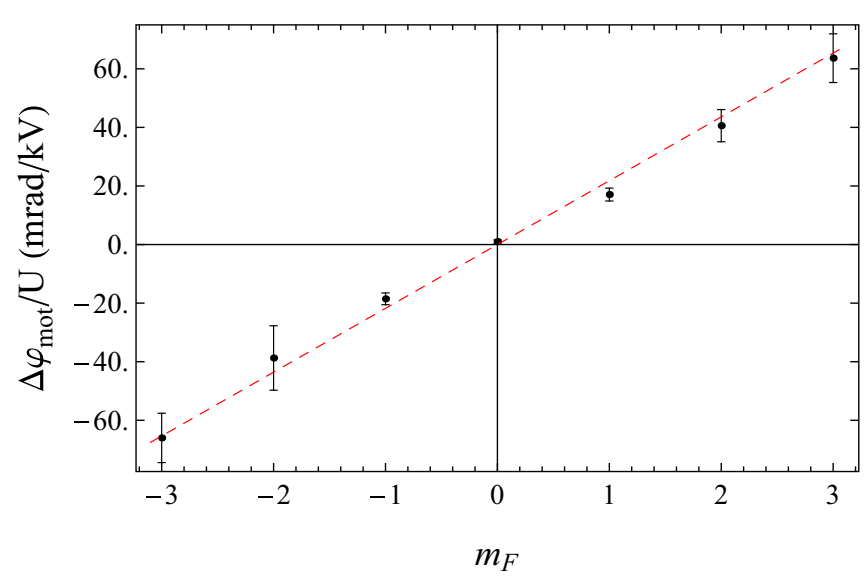

FIG. 12. (Color online) Ramsey signal phase as a function of $m_{F}$ for the seven possible $\Delta m_{F}=0$ hyperfine transitions [each point is the result of a linear fit of the phase $\Delta \varphi_{\text {mot }}(U)$ given by Eq. (28) as a function of the applied voltage $U$ ].

\section{Stark shifts from microwave tracking of the resonance frequency}

Unfortunately, Stark shift measurements using the frequency-tracking method (Fig. 7) described in Sec. IIID were made prior to calibrating the high-voltage (HV) voltmeter. A feedback loop controlling the synthesized microwave frequency $v_{\mu \mathrm{w}}$ was used to lock the latter to the zero crossing of the dispersive central Ramsey fringe (Fig. 2). For each voltage applied to the electric-field generating electrodes, the feedback signal was recorded on a digital oscilloscope for $600 \mathrm{~s}$. Figure 13 shows an example of such a time series.

Stark shift measurements were made for two different relative orientations of the electric and magnetic fields, viz., $\overrightarrow{\mathcal{E}} \perp$ $\overrightarrow{\mathcal{B}}$, corresponding to $f(\theta=\pi / 2)=-1$, and $\overrightarrow{\mathcal{E}} \| \overrightarrow{\mathcal{B}}$, equivalent to $f(\theta=0)=2$. Measurements with the former configuration used $\sigma^{+}-\sigma^{+}$polarized components of the bichromatic optical field, while their polarization was $\pi-\pi$ (with respect to $\overrightarrow{\mathcal{B}}$ ) in the latter configuration. The dependence of the central fringe's

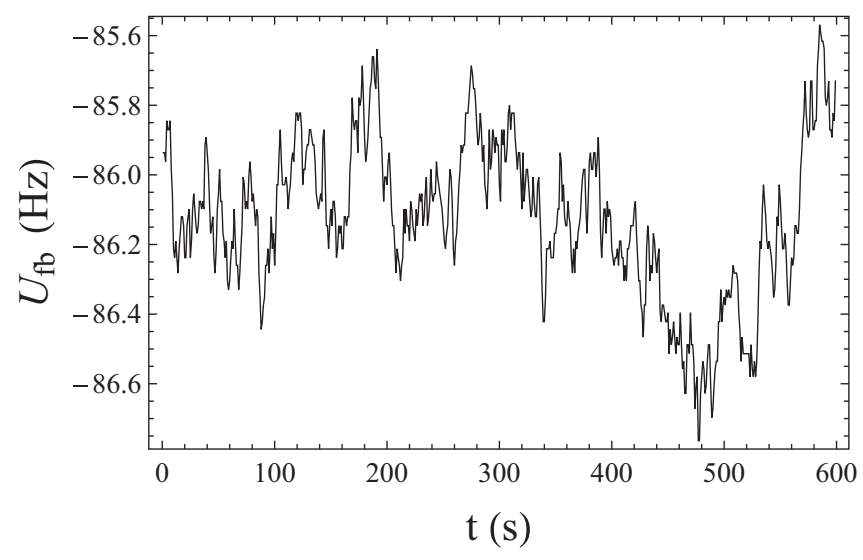

FIG. 13. Typical feedback signal $U_{\mathrm{fb}}$ (calibrated in frequency units) recorded with the microwave frequency locked to the Ramsey fringe center with a voltage of $-4.066 \mathrm{kV} / \mathrm{cm}$. The origin of the ordinate is the frequency generated by the microwave generator, corresponding here to $9.192631770 \mathrm{GHz}$. 


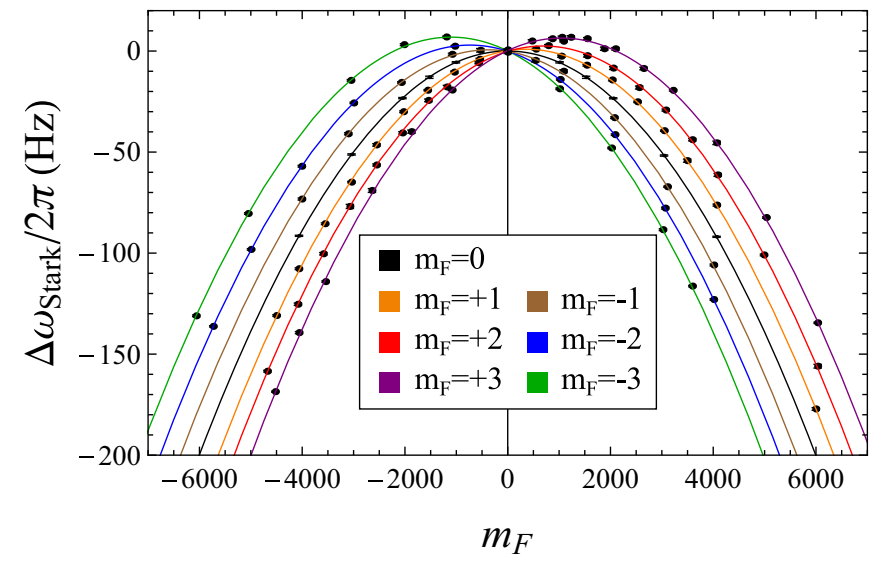

FIG. 14. (Color online) Stark frequency shift of all seven $\Delta m_{F}=0$ hyperfine transitions in the $\overrightarrow{\mathcal{E}} \perp \overrightarrow{\mathcal{B}}$ configuration with $\sigma^{+}-\sigma^{+}$polarized optical fields. The lowest $m_{F}$ value corresponds to the leftmost curve and $m_{F}$ is increasing from left to right. The fitting functions are polynomials of second order in $U$. The lateral displacement of the parabolas is due to the motional magnetic field, while their curvature results from the Stark interaction proper.

shift on the applied electrode voltage $U$ was recorded for all individual $\Delta m_{F}=0$ hyperfine transitions in both field configurations. The data were fitted with a second-order polynomial of the form $\Delta \omega_{\text {Stark }} / 2 \pi=c_{0}+c_{1} U+c_{2} U^{2}$. Figure 14 presents data and the parabolic fit curves for all hyperfine transitions in the $\overrightarrow{\mathcal{E}} \perp \overrightarrow{\mathcal{B}}$ configuration. The $m_{F}$ dependence of the fit parameters $c_{1}$ for both configurations is shown in Fig. 15 and numerical values of all parameters $c_{i}$ are given in Tables III and IV. Note that the $m_{F}=0 \rightarrow m_{F^{\prime}}=0$ transition is forbidden in the $\overrightarrow{\mathcal{E}} \| \overrightarrow{\mathcal{B}}$ configuration.

No physical significance can be attributed to the constant $c_{0}$, which is indeed consistent with zero. The linear coefficient $c_{1}$ is due to the motional magnetic-field-induced phase shift $\Delta \varphi_{\text {mot }}$

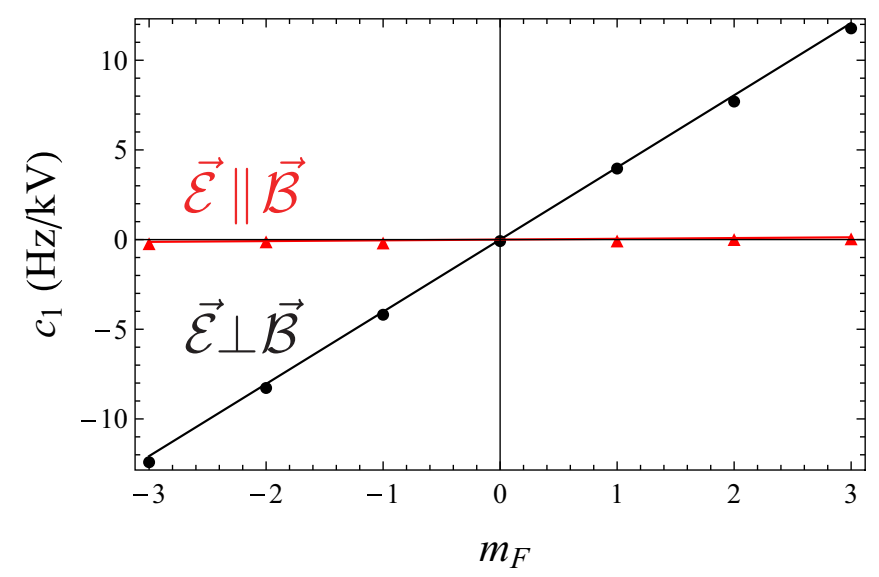

FIG. 15. (Color online) $m_{F}$ dependence of the fit coefficients $c_{1}$ representing the linear (motional field-induced) Stark shift of the $F=3, m_{F} \rightarrow F=4, m_{F}$ transitions. The red triangles show data from Table IV for the $\overrightarrow{\mathcal{E}} \| \overrightarrow{\mathcal{B}}$ configuration, and the black dots show data from Table III for the $\overrightarrow{\mathcal{E}} \perp \overrightarrow{\mathcal{B}}$ configuration. The lines are linear fits to the data. As expected, the motional magnetic field shift vanishes in the $\overrightarrow{\mathcal{E}} \| \overrightarrow{\mathcal{B}}$ configuration.
TABLE III. Polynomial fit parameters for the $\overrightarrow{\mathcal{E}} \perp \overrightarrow{\mathcal{B}}$ configuration.

\begin{tabular}{cccc}
\hline \hline$m_{F} \rightarrow m_{F^{\prime}}$ & $c_{0}(\mathrm{~Hz})$ & $c_{1}(\mathrm{~Hz} / \mathrm{kV})$ & $c_{2}\left[\mathrm{~Hz} /(\mathrm{kV})^{2}\right]$ \\
\hline $0 \rightarrow 0$ & $0.01(9)$ & $-0.036(21)$ & $5.532(4)$ \\
$+1 \rightarrow+1$ & $0.17(14)$ & $4.01(4)$ & $5.593(14)$ \\
$-1 \rightarrow-1$ & $-0.16(24)$ & $-4.14(5)$ & $5.573(27)$ \\
$+2 \rightarrow+2$ & $-0.19(20)$ & $7.74(4)$ & $5.588(15)$ \\
$-2 \rightarrow-2$ & $-0.09(18)$ & $-8.23(5)$ & $5.580(14)$ \\
$+3 \rightarrow+3$ & $0.22(15)$ & $11.83(3)$ & $5.645(9)$ \\
$-3 \rightarrow-3$ & $0.06(18)$ & $-12.37(5)$ & $5.601(15)$ \\
\hline \hline
\end{tabular}

of Eqs. (28), which influences the $\Delta \omega_{\text {Stark }}(U)$ dependence in different ways for the two field configurations. In the $\overrightarrow{\mathcal{E}} \| \overrightarrow{\mathcal{B}}$ configuration, the motional magnetic field $(\approx n T)$ is perpendicular to the applied magnetic field of $\sim 4 \mu \mathrm{T}$, so that the motional field adds quadratically to the latter, thus giving a negligible contribution. As expected, the fitted $c_{1}$ coefficients for $\overrightarrow{\mathcal{E}} \| \overrightarrow{\mathcal{B}}$ are compatible with zero within three standard deviations. In the $\overrightarrow{\mathcal{E}} \perp \overrightarrow{\mathcal{B}}$ configuration, on the other hand, the motional magnetic field is directed along the applied magnetic field, and thus increases or decreases the latter directly. Being a Zeeman effect, the motional field effect is expected to be proportional to $m_{F}$, a feature that is well obeyed by the experimental data shown in Fig. 15.

The fitted $c_{2}$ coefficients are related to the Stark polarizabilities $\alpha\left(m_{F}\right)$ and the polarizability of interest $\alpha_{0}^{(3)}$ via

$$
\begin{aligned}
\frac{\alpha\left(m_{F}\right)}{2} & =-c_{2}\left(d^{2}\right)_{\mathrm{eff}} \\
& =\left[\frac{8}{7} \alpha_{0}^{(3)}+\frac{4}{7} f(\theta) \alpha_{2}^{(3)}\right]-\frac{3}{28} f(\theta) \alpha_{2}^{(3)} m_{F}^{2},
\end{aligned}
$$

where $\alpha_{\mathrm{k}}^{(3)}=\alpha_{\mathrm{k}}^{(3)}(F=4)$, and where $f(\theta)=+2$ and -1 for the $\overrightarrow{\mathcal{E}} \| \overrightarrow{\mathcal{B}}$ and $\overrightarrow{\mathcal{E}} \perp \overrightarrow{\mathcal{B}}$ configurations, respectively. The polarizability $\alpha\left(m_{F}\right)=-2 \Delta v_{\text {Stark }}\left(m_{F}\right) / \mathcal{E}^{2}$, extracted from the measured Stark shifts $\Delta v_{\text {Stark }}\left(m_{F}\right)$ using $\mathcal{E}^{2}=U^{2} /\left(d^{2}\right)_{\text {eff }}$, has $m_{F}$-independent and $m_{F}$-dependent contributions from the third-order polarizabilities $\alpha_{0}^{(3)}$ and $\alpha_{2}^{(3)}$, with $\alpha_{2}^{(3)}$ being $\sim 2$ orders of magnitude smaller than $\alpha_{0}^{(3)}$.

The experimental values of $\alpha\left(m_{F}\right) / 2$ inferred from the fitted coefficients $c_{2}$ following Eq. (35a) are shown in Fig. 16, together with fitted curves, and exhibit the anticipated quadratic $m_{F}$ dependence, with curvatures in the ratio $(-1): 2$,

TABLE IV. Polynomial fit parameters for the $\overrightarrow{\mathcal{E}} \| \overrightarrow{\mathcal{B}}$ configuration. The $m_{F}=0 \rightarrow m_{F^{\prime}}=0$ clock transition is forbidden in this configuration.

\begin{tabular}{crrr}
\hline \hline$m_{F} \rightarrow m_{F^{\prime}}$ & $c_{0}(\mathrm{~Hz})$ & $c_{1}(\mathrm{~Hz} / \mathrm{kV})$ & $c_{2}\left[\mathrm{~Hz} /(\mathrm{kV})^{2}\right]$ \\
\hline $0 \rightarrow 0$ & & & \\
$+1 \rightarrow+1$ & $0.55(45)$ & $-0.011(6)$ & $5.609(17)$ \\
$-1 \rightarrow-1$ & $1.09(37)$ & $-0.120(50)$ & $5.605(8)$ \\
$+2 \rightarrow+2$ & $0.02(40)$ & $0.068(47)$ & $5.577(14)$ \\
$-2 \rightarrow-2$ & $-0.11(42)$ & $-0.061(57)$ & $5.573(17)$ \\
$+3 \rightarrow+3$ & $-0.08(52)$ & $0.110(70)$ & $5.532(23)$ \\
$-3 \rightarrow-3$ & $-0.46(52)$ & $-0.160(60)$ & $5.544(24)$ \\
\hline \hline
\end{tabular}




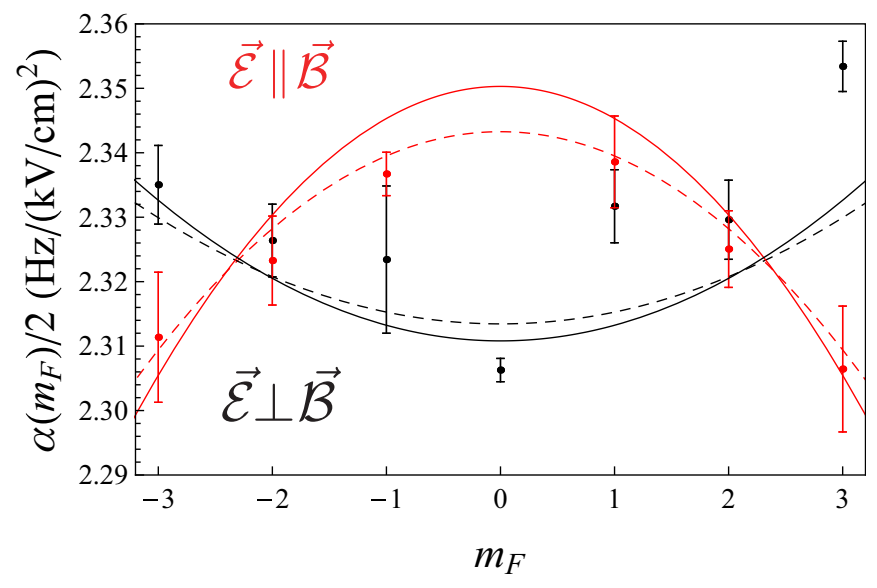

FIG. 16. (Color online) $m_{F}$ dependence of the differential Stark polarizability $\alpha\left(m_{F}\right) / 2$ from Ramsey fringe frequency-tracking experiments using two field configurations, together with fits of Eq. (35) made to all of the data at once. Red represents $f(\theta)=+2$ data and black represents $f(\theta)=-1$. The solid lines are fits made with both $\alpha_{0}^{(3)}$ and $\alpha_{2}^{(3)}$ as free parameters. The dashed lines are fits with $\alpha_{2}^{(3)}$ fixed to the best existing literature value.

as predicted by the $f(\theta)$ dependence. Note that based on Eq. (35b), the fitted curves are expected to intersect at the "fictitious" $m_{F}$ value of $m_{F}^{*}=\sqrt{16 / 3} \approx 2.31$, for which $\alpha\left(m_{F}^{*}\right) / 2=8 \alpha_{0}^{(3)}(F=4) / 7=-k_{s}$.

We have taken two approaches for fitting Eq. (35b) to the data of Fig. 16. In a first approach, we have left both $\alpha_{0}^{(3)}(F=4)$ and $\alpha_{2}^{(3)}(F=4)$ as free parameters and, in a second approach, we have left only $\alpha_{0}^{(3)}(F=4)$ as a free parameter, by fixing $\alpha_{2}^{(3)}(F=4)$ to

$$
\alpha_{2}^{(3)}(F=4)=-0.0351(16) \mathrm{Hz} /(\mathrm{kV} / \mathrm{cm})^{2},
$$

taken as the weighted average of published measurements $[19,20,29]$. In both cases, all data were fit simultaneously. The two fit methods yield the same value for the scalar polarizability,

$$
\begin{aligned}
\alpha_{0}^{(3)}(F=4) & =2.033(1)_{\text {stat }}(22)_{\text {syst }} \mathrm{Hz} /(\mathrm{kV} / \mathrm{cm})^{2} \\
& =2.033(22) \mathrm{Hz} /(\mathrm{kV} / \mathrm{cm})^{2},
\end{aligned}
$$

and the scalar Stark shift parameter

$$
\begin{aligned}
k_{s} & =-2.323(1)_{\text {stat }}(25)_{\text {syst }} \mathrm{Hz} /(\mathrm{kV} / \mathrm{cm})^{2} \\
& =-2.323(25) \mathrm{Hz} /(\mathrm{kV} / \mathrm{cm})^{2},
\end{aligned}
$$

respectively, where the errors in Eqs. (37b) and (38b) represent the squared sums of statistical and systematic errors, respectively.

When fitted as a free parameter, we obtain a tensor polarizability

$$
\alpha_{2}^{(3)}(F=4)=-0.046(4) \mathrm{Hz} /(\mathrm{kV} / \mathrm{cm})^{2}
$$

that agrees with the literature average within two standard deviations.

The relative statistical uncertainty of $\alpha_{0}^{(3)}(F=4)$ obtained by the frequency-tracking method is rather small, $\sim 5 \times 10^{-4}$. However, the systematic uncertainty on the polarizability in the fringe-tracking experiment is dominated by imprecise knowledge of the voltage drop across the field electrodes. In those early experiments, the $200 \mathrm{M} \Omega$ protection resistor was in series with the field-producing capacitor, but the voltage was measured directly at the supply output. Any leakage current across the field plates will thus lead to a voltage drop over the protection resistor, thereby lowering the effective voltage applied to the electrodes. We estimate the systematic field uncertainty due to this unfortunate configuration as follows: Based on the current $(\sim 1 \mu \mathrm{A})$ drawn from the power supply and the digital high-voltage voltmeter's $6.5 \mathrm{G} \Omega$ internal resistance, we estimate the uncertainty on the electrode voltage $U$ to be $\sim 5 \times 10^{-3}$. Quadratically adding the latter uncertainty and the $\sim 5 \times 10^{-3}$ uncertainty of the field calibration constant $\left(d^{2}\right)_{\text {eff }}$ yields the systematic errors of Eqs. (37) and (38).

\section{SUMMARY AND CONCLUSION}

We have used two separate methods to measure the differential third-order electric polarizability of the Cs ground-state hyperfine levels, from which we infer the third-order scalar polarizability $\alpha_{0}^{(3)}(F=4)$ of the $F=4$ state and the scalar Stark shift coefficient $k_{s}$, which are related by

$$
k_{s}=-\frac{8}{7} \alpha_{0}^{(3)}(F=4) \text {. }
$$

The result obtained by the fringe-tracking method has a very small statistical error, but suffers from a large systematic uncertainty because of imprecisions in the voltage measurement. The $\alpha_{0}^{(3)}(F=4)$ value obtained by the Fourier analysis method, on the other hand, has comparable statistical and systematic errors. The individual results and their average value are presented in Fig. 17, together with past experimental $[4,5,30,31]$ and theoretical $[9,10]$ values. For a comparison with more previous results, we refer the reader to Fig. 3 of Ref. [4].

The weighted average of our two independent measurements, expressed in terms of the scalar Stark shift coefficient

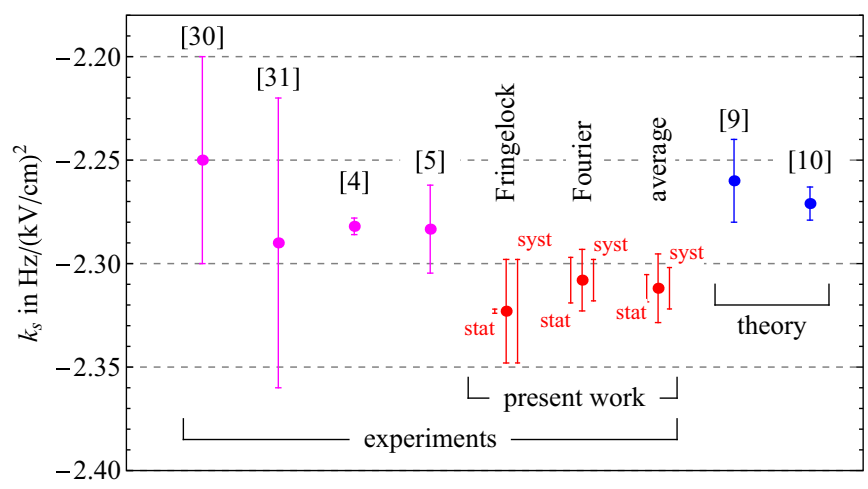

FIG. 17. (Color online) Comparison of results from present work with previous experimental and theoretical values (see reference numbers in brackets in figure) for the Cs clock transition's scalar Stark shift coefficient $k_{s}$. The statistical and systematic uncertainty intervals of our results are shown on the left and right, respectively, of the experimental data points. The errors on our data points were derived from those errors as discussed in the text. Note that the value $k_{s}=2.050(40) \mathrm{Hz} /(\mathrm{kV} / \mathrm{cm})^{2}$ of Godone et al. [6] is far off scale of the present plot. 
$k_{s}$, yields

$$
\begin{aligned}
k_{s} & =-\frac{8}{7} \alpha_{0}^{(3)}(F=4) \\
& =-2.312(7)_{\text {stat }}(10)_{\text {syst }} \mathrm{Hz} /(\mathrm{kV} / \mathrm{cm})^{2} .
\end{aligned}
$$

The (quadratically) combined statistical and systematic errors of the individual measurements given in Eqs. (34b) and (38b), respectively, were used as weights to derive the final value. The statistical error on the final value is the statistical error of the mean. The systematic error of the final value represents the systematic uncertainty of the Fourier analysis data, with the (larger) systematic error of the fringe-tracking data playing a subordinate role because of the reduced contribution of those data to the final result,

$$
k_{s}=-2.312(17) \mathrm{Hz} /(\mathrm{kV} / \mathrm{cm})^{2},
$$

where we have taken the conservative approach by (linearly) adding the statistical and systematic errors of (41).

The $k_{s}$ value of (42) is equivalent to

$$
\begin{aligned}
\alpha_{0}^{(3)}(F=4) & =2.023(6)_{\mathrm{stat}}(9)_{\mathrm{syst}} \mathrm{Hz} /(\mathrm{kV} / \mathrm{cm})^{2} \\
& =2.023(15) \mathrm{Hz} /(\mathrm{kV} / \mathrm{cm})^{2}
\end{aligned}
$$

and

$$
\begin{aligned}
\beta & =\frac{k_{s}}{v_{00}}\left(831.9 \frac{\mathrm{V}}{\mathrm{m}}\right)^{2} \\
& =-1.7406(53)_{\mathrm{stat}}(75)_{\mathrm{syst}} \times 10^{-14}
\end{aligned}
$$

Our result has a relative error of $0.7 \%$ and is to be compared to the most precise experimental value published to date [4],

$$
k_{s}=2.282(4) \mathrm{Hz} /(\mathrm{kV} / \mathrm{cm})^{2},
$$

with a relative uncertainty below $0.2 \%$ that testifies to the remarkable control of the field integral in that experiment. Our result differs by $\sim 2$ standard deviations from that measurement and from theoretical predictions, and can be considered to be in agreement with those results.

\section{ACKNOWLEDGMENTS}

This work was funded by the Swiss National Science Foundation, Grants No. 200020-126499 and No. 200021117841. Support from the University of Fribourg Fonds de Recherche and the Physics Department's mechanical and electronic workshops is acknowledged. Dr. Z. Andjelić and ABB Corporate Research, Baden, Switzerland are thanked for their generous donation of time and resources for the field modeling.
[1] W. M. Itano, L. L. Lewis, and D. J. Wineland, Phys. Rev. A 25, 1233 (1982).

[2] R. Wynands and S. Weyers, Metrologia 42, S64 (2005).

[3] J. Mitroy, M. S. Safronova, and C. W. Clark, J. Phys. B 43, 202001 (2010).

[4] P. Rosenbusch, S. Zhang, and A. Clairon, in IEEE International 2007 Joint Frequency Control Symposium with the 21st European Frequency and Time Forum (IEEE, Piscataway, NJ, 2007), Vol. 29, pp. 1060.

[5] S. R. Jefferts, T. P. Heavner, T. E. Parker, J. H. Shirley, E. A. Donley, N. Ashby, F. Levi, D. Calonico, and G. A. Costanzo, Phys. Rev. Lett. 112, 050801 (2014).

[6] A. Godone, D. Calonico, F. Levi, S. Micalizio, and C. Calosso, Phys. Rev. A 71, 063401 (2005).

[7] E. Simon, P. Laurent, and A. Clairon, Phys. Rev. A 57, 436 (1998).

[8] P. Rosenbusch, S. Ghezali, V. A. Dzuba, V. V. Flambaum, K. Beloy, and A. Derevianko, Phys. Rev. A 79, 013404 (2009).

[9] E. J. Angstmann, V. A. Dzuba, and V. V. Flambaum, Phys. Rev. Lett. 97, 040802 (2006).

[10] K. Beloy, U. I. Safronova, and A. Derevianko, Phys. Rev. Lett. 97, 040801 (2006).

[11] J. H. Shirley, IEEE Trans. Instrum. Meas. 46, 117 (1997).

[12] G. Di Domenico, L. Devenoges, A. Stefanov, A. Joyet, and P. Thomann, Eur. Phys. J. Appl. Phys. 56, 11001 (2011).

[13] J. R. P. Angel and P. G. H. Sandars, Proc. R. Soc. London, A 305, 125 (1968).

[14] S. Ulzega, Ph.D. thesis, University of Fribourg, Switzerland, 2006.

[15] S. Ulzega, A. Hofer, P. Moroshkin, and A. Weis, Europhys. Lett. 76, 1074 (2006); see Errata in Ref. [16].

[16] S. Ulzega, A. Hofer, P. Moroshkin, and A. Weis, Europhys. Lett. 78, 69901 (2007).

[17] P. G. H. Sandars, Proc. Phys. Soc. 92, 857 (1967).
[18] E. J. Angstmann, V. A. Dzuba, and V. V. Flambaum, Phys. Rev. A 74, 023405 (2006).

[19] J. P. Carrico, A. Adler, M. R. Baker, S. Legowski, E. Lipworth, P. G. H. Sandars, T. S. Stein, and C. Weisskopf, Phys. Rev. 170, 64 (1968).

[20] H. Gould, E. Lipworth, and M. C. Weisskopf, Phys. Rev. 188, 24 (1969).

[21] J.-L. Robyr, P. Knowles, and A. Weis, IEEE Trans. Ultrason. Ferroelectr. Freq. Control 57, 613 (2010).

[22] J.-L. Robyr, P. Knowles, and A. Weis, in IEEE International 2011 Joint Frequency Control Symposium with the 24th European Frequency and Time Forum (IEEE, San Francisco, 2011), pp. $1-4$.

[23] J.-L. Robyr, Ph.D. thesis, University of Fribourg, Switzerland, 2011.

[24] N. F. Ramsey, Molecular Beams (Oxford University Press, London, 1956).

[25] J.-L. Robyr, P. Knowles, and A. Weis, in IEEE International 2009 Joint Frequency Control Symposium with the 22nd European Frequency and Time Forum (IEEE, Besançon, France, 2009), pp. 600-603.

[26] R. D. Swenumson and U. Even, Rev. Sci. Instrum. 52, 559 (1981).

[27] Z. Andjelić, computer code POLOPT, http://www.polopt.com/.

[28] Z. Andjelić, in Domain Decomposition Methods in Science and Engineering XVII, edited by U. Langer, M. Discacciati, D. E. Keyes, O. B. Widlund, and W. Zulehner, Lecture Notes in Computational Science and Engineering Vol. 60 (Springer, Heidelberg, 2008), p. 155 .

[29] C. Ospelkaus, U. Rasbach, and A. Weis, Phys. Rev. A 67, 011402 (2003).

[30] J. R. Mowat, Phys. Rev. A 5, 1059 (1972).

[31] R. D. Haun and J. R. Zacharias, Phys. Rev. 107, 107 (1957). 